\title{
mHealth for HIV Treatment \& Prevention: A Systematic Review of the Literature
}

\author{
Caricia Catalani ${ }^{*}, 1$, William Philbrick ${ }^{2}$, Hamish Fraser $^{3}$, Patricia Mechael $^{2}$ and Dennis M. Israelski ${ }^{1}$ \\ ${ }^{1}$ InSTEDD \& University of California, Berkeley, USA \\ ${ }^{2}$ mHealth Alliance, USA \\ ${ }^{3}$ Partners in Health \& Brigham and Womens Hospital, USA
}

\begin{abstract}
This systematic review assesses the published literature to describe the landscape of mobile health technology (mHealth) for HIV/AIDS and the evidence supporting the use of these tools to address the HIV prevention, care, and treatment cascade. The speed of innovation, broad range of initiatives and tools, and heterogeneity in reporting have made it difficult to uncover and synthesize knowledge on how mHealth tools might be effective in addressing the HIV pandemic.

To do address this gap, a team of reviewers collected literature on the use of mobile technology for HIV/AIDS among health, engineering, and social science literature databases and analyzed a final set of 62 articles. Articles were systematically coded, assessed for scientific rigor, and sorted for HIV programmatic relevance. The review revealed evidence that mHealth tools support HIV programmatic priorities, including: linkage to care, retention in care, and adherence to antiretroviral treatment. In terms of technical features, mHealth tools facilitate alerts and reminders, data collection, direct voice communication, educational messaging, information on demand, and more. Studies were mostly descriptive with a growing number of quasi-experimental and experimental designs. There was a lack of evidence around the use of mHealth tools to address the needs of key populations, including pregnant mothers, sex workers, users of injection drugs, and men who have sex with men.

The science and practice of mHealth for HIV are evolving rapidly, but still in their early stages. Small-scale efforts, pilot projects, and preliminary descriptive studies are advancing and there is a promising trend toward implementing mHealth innovation that is feasible and acceptable within low-resource settings, positive program outcomes, operational improvements, and rigorous study design.
\end{abstract}

Keywords: HIV Treatment, HIV Prevention, information technology, mobile phone, mHealth.

\section{INTRODUCTION}

Today, mobile health information technologies are being deployed around the world to address a spectrum of challenges from disease surveillance to health systems strengthening to health education [1]. Health professionals, policymakers, and technologists alike have been inspired by the promise of eHealth and mHealth technologies during the present wave of innovation. The speed of innovation, broad range of pilot initiatives and tools, and inconsistencies in reporting have made it difficult to uncover and synthesize knowledge on how these tools might be effective in addressing global health crises such as the HIV pandemic.

For the first time since the dawn of the epidemic, major clinical trials have confirmed the effectiveness of several prevention strategies [2-5]. Pledges by multinational donors to achieve universal access to HIV treatment, care, and support [6] and the stated strategic goal of the President's Emergency Plan for AIDS Relief (PEPfAR) of achieving

*Address correspondence to this author at the University of California, Berkeley \& InSTEDD, USA; Tel: (415) 992- 7947;

E-mails: Caricia@instedd.org, catalani@berkeley.edu
"AIDS free generation" has led to optimism with regard to the potential for elimination of HIV globally [7]. To achieve universal access to treatment, national and local programs must expand access to HIV care, strengthen health systems, improve quality of care delivered, reduce costs, and address barriers to access at every stage of the HIV treatment cascade [8-11]. Early reports on mHealth suggest that mobile health technologies may help to address these programmatic needs [12].

mHealth (mobile health) can be defined as the practice of medical and public health via mobile communication devices $[13,14]$. Although explicitly focused on the use of mobile devices, the full promise of mHealth may require understanding it within in the broader context of eHealth defined as the transfer of health resources and health care my electronic means [15] - and comprehensive health information systems. mHealth tools have been particularly useful in low- and middle-income countries (LMICs), where mobile phones and similar devices have the added advantage of extended battery life, simple interface for technology users, voice operability, cellular connectivity, and relative affordability. 
This systematic review describes the overall landscape of mHealth for HIV/AIDS as reflected in the published literature and evidence related to the use of mHealth to address specific programmatic requirements along the cascade of HIV prevention, care, and treatment as reflected in the research literature.

\section{METHODS}

Initially, a search of all published articles referring to mHealth was conducted. We searched for the following terms within title, abstract, or author keywords using this logic: mHealth OR ((handheld computers OR mobile device OR cellular phone OR mobile phone) AND health). We limited the search to articles published in English during the past ten years, from January 2001 to December 2011. The term handheld computer captured a variety of types of devices, including tablet devices such as iPads, personal digital assistants (PDAs), and devices for global positioning systems (GPS). To capture this multidisciplinary field, we searched among health, engineering, technology, and social science databases: Pubmed/Medline, Scopus, Embase, Cochrane, CABI Global Health, Compendex, Web of Science, INSPEC, Popline, WHO Technical Reports, and Google Scholar. Finally, we searched within the collection of unique mHealth articles (as depicted in Fig. 1) to identify only those referring to HIV or AIDS within the title, abstract, or author keywords.

To categorize and assess the literature, three independent reviewers systematically coded each article to identify descriptions of the following topics: methods, design, implementation science category, rigor, purpose of technology, theoretical framework, location, population served, community engagement/participation, stage of cascade, impact, findings, and conclusions. Finally, coded information was gathered and summarized for each article by each reviewer. If there was any disagreement between all three reviewers' code summaries, a senior researcher fully reviewed the article and code to settle the discrepancy with their final determination. This process resulted in the article descriptions in Tables $\mathbf{1}$ and $\mathbf{2}$.

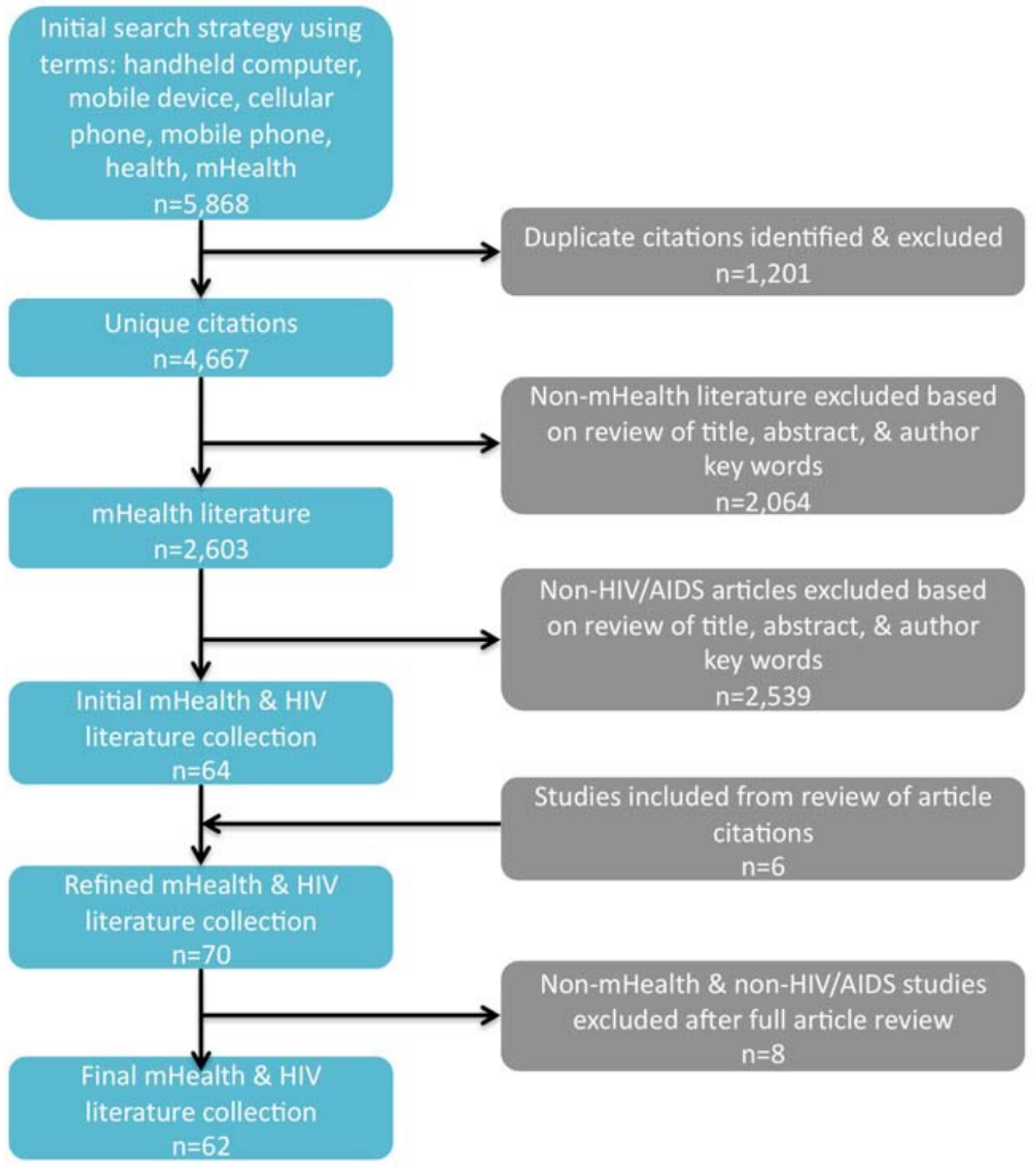

Fig. (1). PRISMA flow diagram. 
Table 1. Non-Research Literature

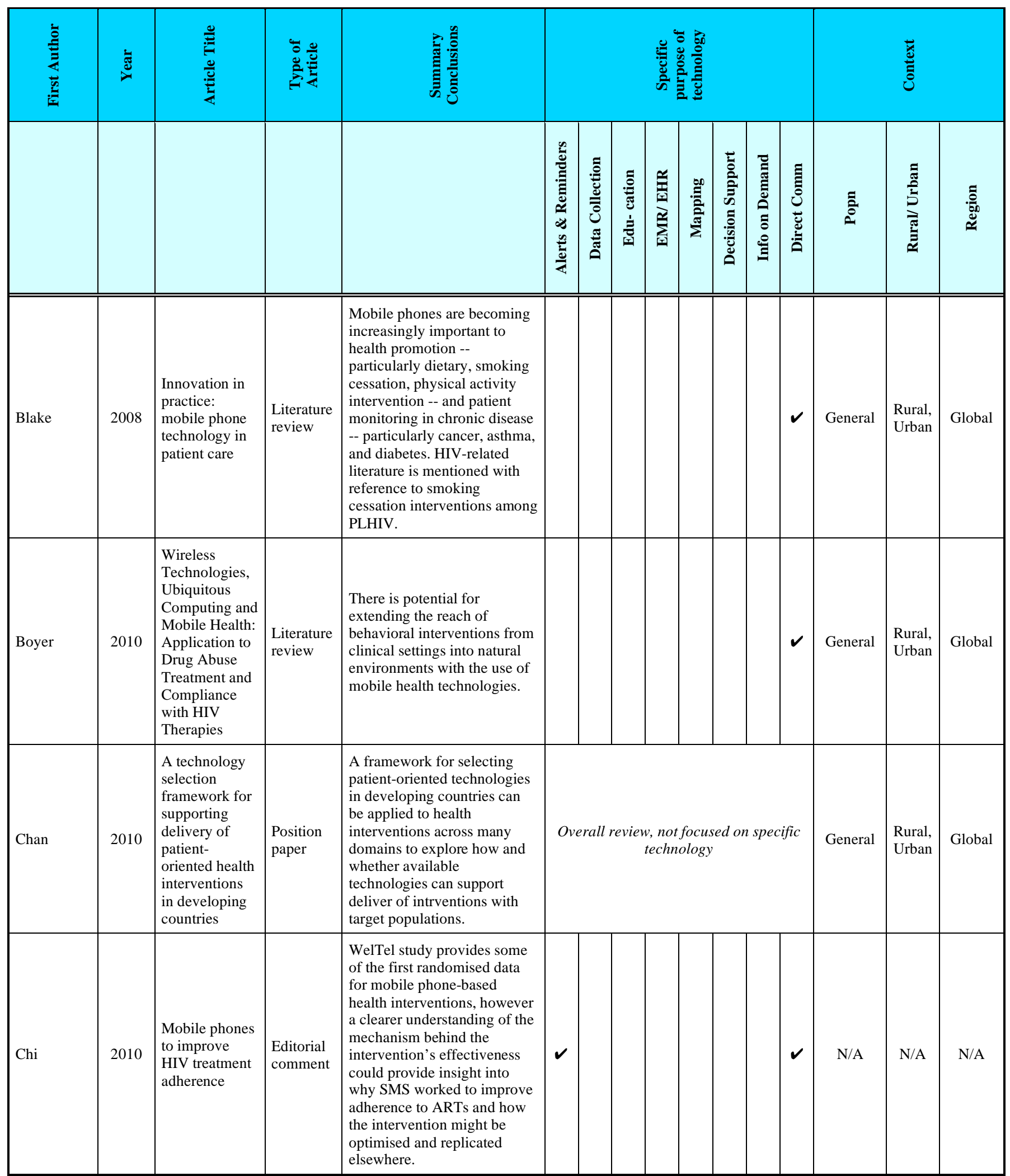




\begin{tabular}{|c|c|c|c|c|c|c|c|c|c|c|c|c|c|c|c|}
\hline \multirow[t]{2}{*}{ 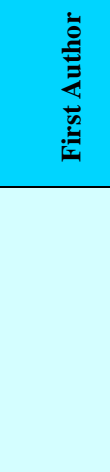 } & \multirow[t]{2}{*}{ 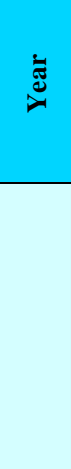 } & \multirow[t]{2}{*}{ 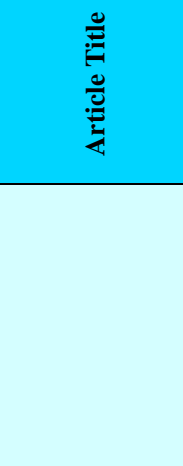 } & \multirow[t]{2}{*}{ 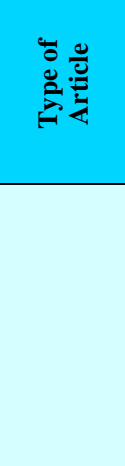 } & \multirow[t]{2}{*}{ 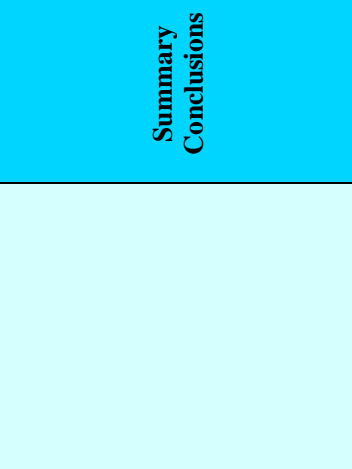 } & \multicolumn{8}{|c|}{ 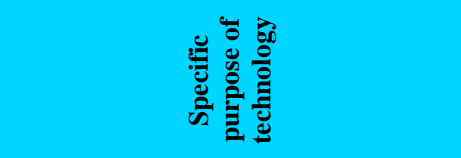 } & \multicolumn{3}{|c|}{ ن⿺辶大 } \\
\hline & & & & & 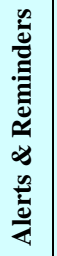 & 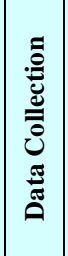 & 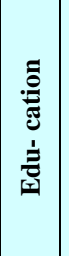 & 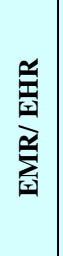 & 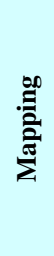 & 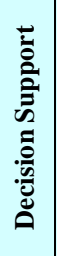 & 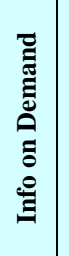 & 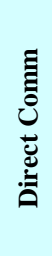 & 气ิ & 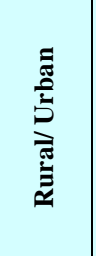 & 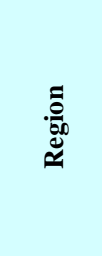 \\
\hline Chiasson & 2010 & $\begin{array}{l}\text { HIV } \\
\text { Prevention and } \\
\text { Care in the } \\
\text { Digital Age }\end{array}$ & $\begin{array}{l}\text { Position } \\
\text { paper }\end{array}$ & $\begin{array}{l}\text { HIV prevention and care } \\
\text { programs using digital media } \\
\text { have great potential to cost- } \\
\text { effectively meet the complex } \\
\text { needs of diverse and often } \\
\text { underserved populations } \\
\text { living with or at high risk of } \\
\text { HIV. }\end{array}$ & \multicolumn{8}{|c|}{$\begin{array}{l}\text { Overall review, not focused on specific } \\
\text { technology }\end{array}$} & $\begin{array}{l}\text { PLWHA, } \\
\text { Provider } \\
\text { s. MSM }\end{array}$ & $\begin{array}{l}\text { Rural, } \\
\text { Urban }\end{array}$ & $\begin{array}{c}\text { North } \\
\text { Americ } \\
\text { a }\end{array}$ \\
\hline Curioso & 2010 & $\begin{array}{l}\text { Enhancing 'M- } \\
\text { Health' With } \\
\text { South- To- } \\
\text { South } \\
\text { Collaborations }\end{array}$ & $\begin{array}{l}\text { Position } \\
\text { paper }\end{array}$ & $\begin{array}{l}\text { South-to-South partnerships } \\
\text { are key to maximizing the } \\
\text { benefits of mHealth } \\
\text { technologies. }\end{array}$ & \multicolumn{8}{|c|}{$\begin{array}{c}\text { Overall review, not focused on specific } \\
\text { technology }\end{array}$} & N/A & N/A & N/A \\
\hline de Costa & 2010 & $\begin{array}{l}\text { Design of a } \\
\text { randomized } \\
\text { trial to } \\
\text { evaluate the } \\
\text { influence of } \\
\text { mobile phone } \\
\text { reminders on } \\
\text { adherence to } \\
\text { first line } \\
\text { antiretroviral } \\
\text { treatment in } \\
\text { South India - } \\
\text { the HIVIND } \\
\text { study protocol }\end{array}$ & $\begin{array}{l}\mathrm{RCT} \\
\text { descriptio } \\
\mathrm{n}\end{array}$ & N/A & $\checkmark$ & & & & & & & & PLWHA & $\begin{array}{l}\text { Rural, } \\
\text { Urban }\end{array}$ & Asia \\
\hline Driscoll & 2001 & $\begin{array}{l}\text { HIV/AIDS and } \\
\text { information } \\
\text { and } \\
\text { communicatio } \\
\mathrm{n} \text { technologies }\end{array}$ & $\begin{array}{l}\text { Policy } \\
\text { analysis }\end{array}$ & $\begin{array}{l}\text { IDRC, either alone, or as a } \\
\text { member of the UNDP- } \\
\text { initiated coalition can ensure } \\
\text { the responsible and ethical } \\
\text { introduction and } \\
\text { implementation of ICTs when } \\
\text { defining programs and } \\
\text { policies. }\end{array}$ & \multicolumn{8}{|c|}{$\begin{array}{c}\text { Overall review, not focused on specific } \\
\text { technology }\end{array}$} & N/A & N/A & N/A \\
\hline Evans & 2007 & $\begin{array}{l}\text { A randomized } \\
\text { trial evaluating } \\
\text { Prosaptide for } \\
\text { HIV- } \\
\text { associated } \\
\text { sensory } \\
\text { neuropathies: } \\
\text { use of an } \\
\text { electronic } \\
\text { diary to record } \\
\text { neuropathic } \\
\text { pain. }\end{array}$ & $\begin{array}{l}\text { RCT, } \\
\text { pharma } \\
\text { efficacy } \\
\text { trial }\end{array}$ & $\begin{array}{l}\text { Electronic diary is a viable } \\
\text { method for collecting patient } \\
\text { information, particularly in } \\
\text { regards to pain measurement, } \\
\text { for a short period of time } \\
\text { among diverse participants. } \\
\text { However, study found that 6- } \\
\text { week treatment with PRO was } \\
\text { safe but not effective at } \\
\text { reducing HIV-associated } \\
\text { neuropathic pain. }\end{array}$ & & $\checkmark$ & & & & & & & PLWHA & $\mathrm{N} / \mathrm{R}$ & $\begin{array}{c}\text { North } \\
\text { Americ } \\
\mathrm{a}\end{array}$ \\
\hline Fahey & 2003 & $\begin{array}{l}\text { Reminding } \\
\text { patients by text } \\
\text { message text } \\
\text { reminders }\end{array}$ & $\begin{array}{l}\text { Editorial } \\
\text { Comment }\end{array}$ & $\begin{array}{l}\text { Patient reminder systems } \\
\text { might be financially } \\
\text { supported through reverse } \\
\text { billing. }\end{array}$ & $\checkmark$ & & & & & & & & N/A & N/A & N/A \\
\hline
\end{tabular}




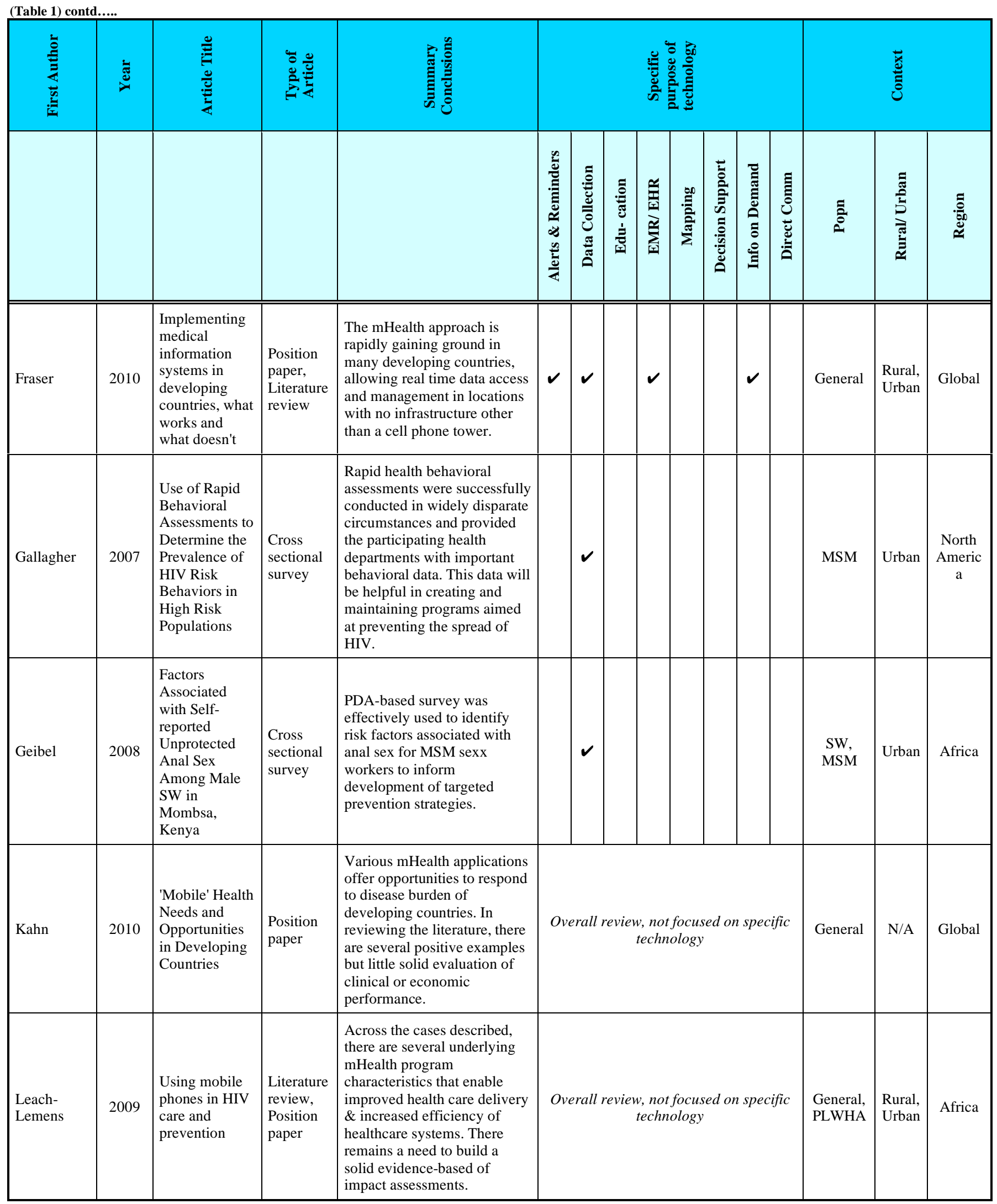




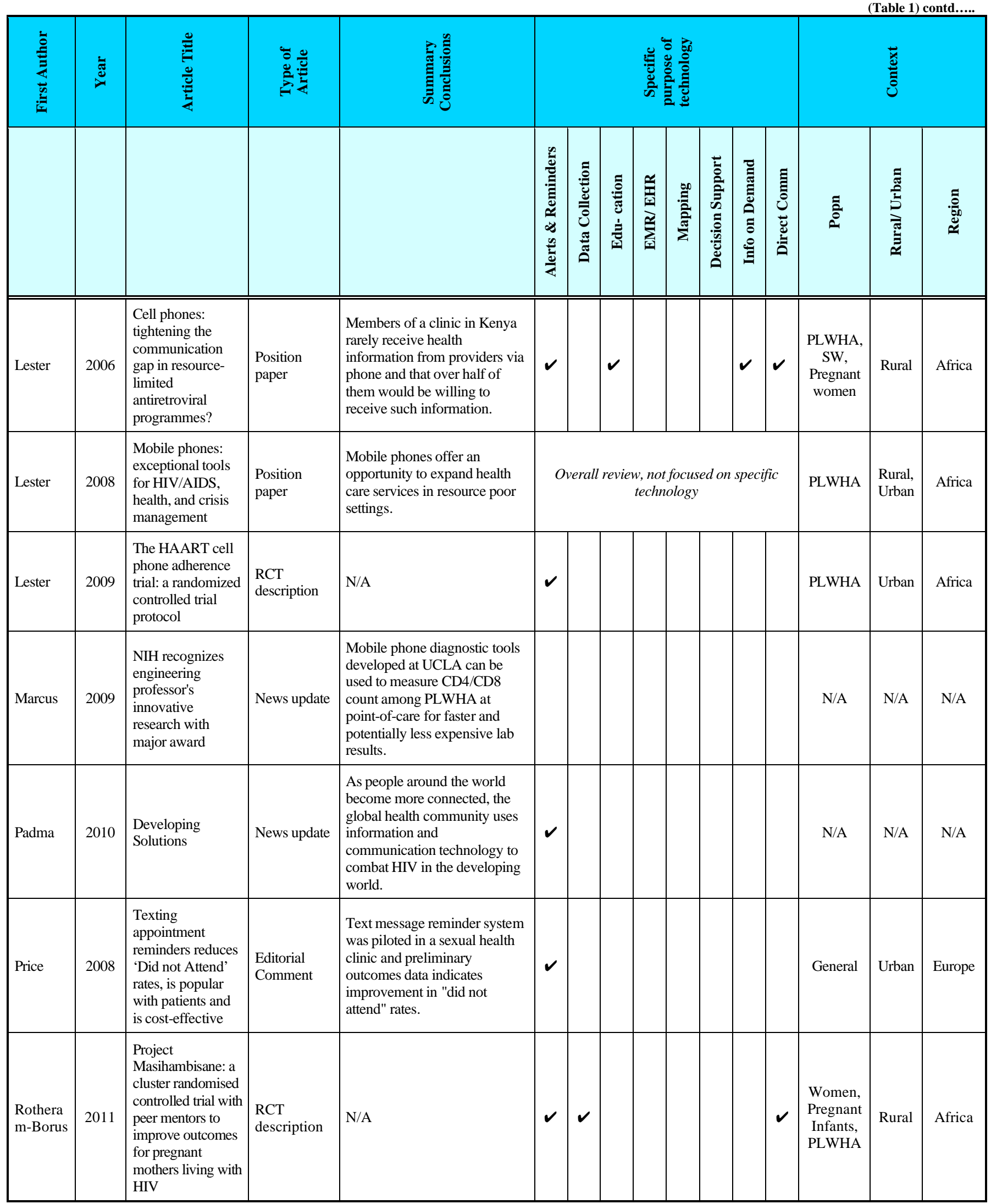




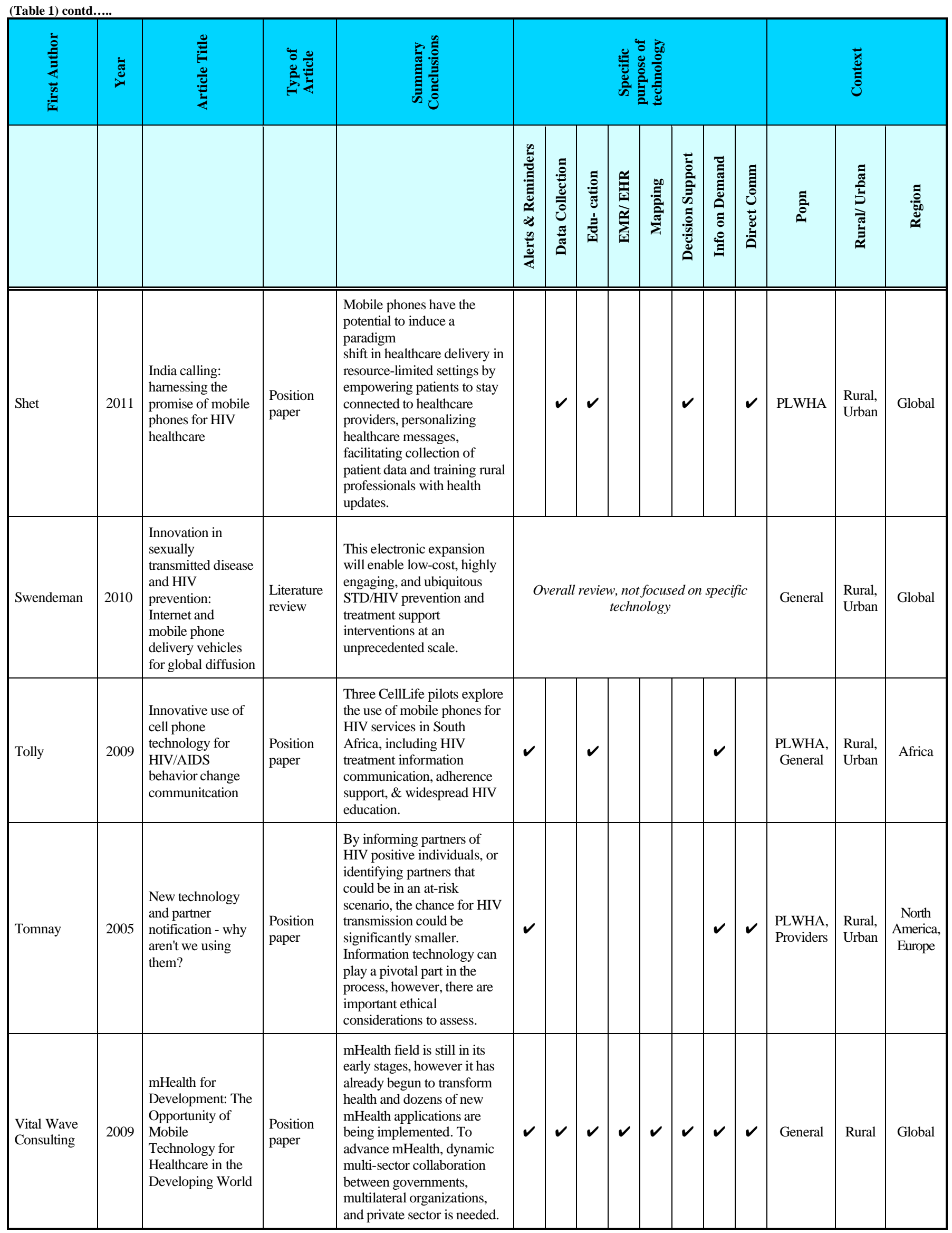


Table 2. Research Literature

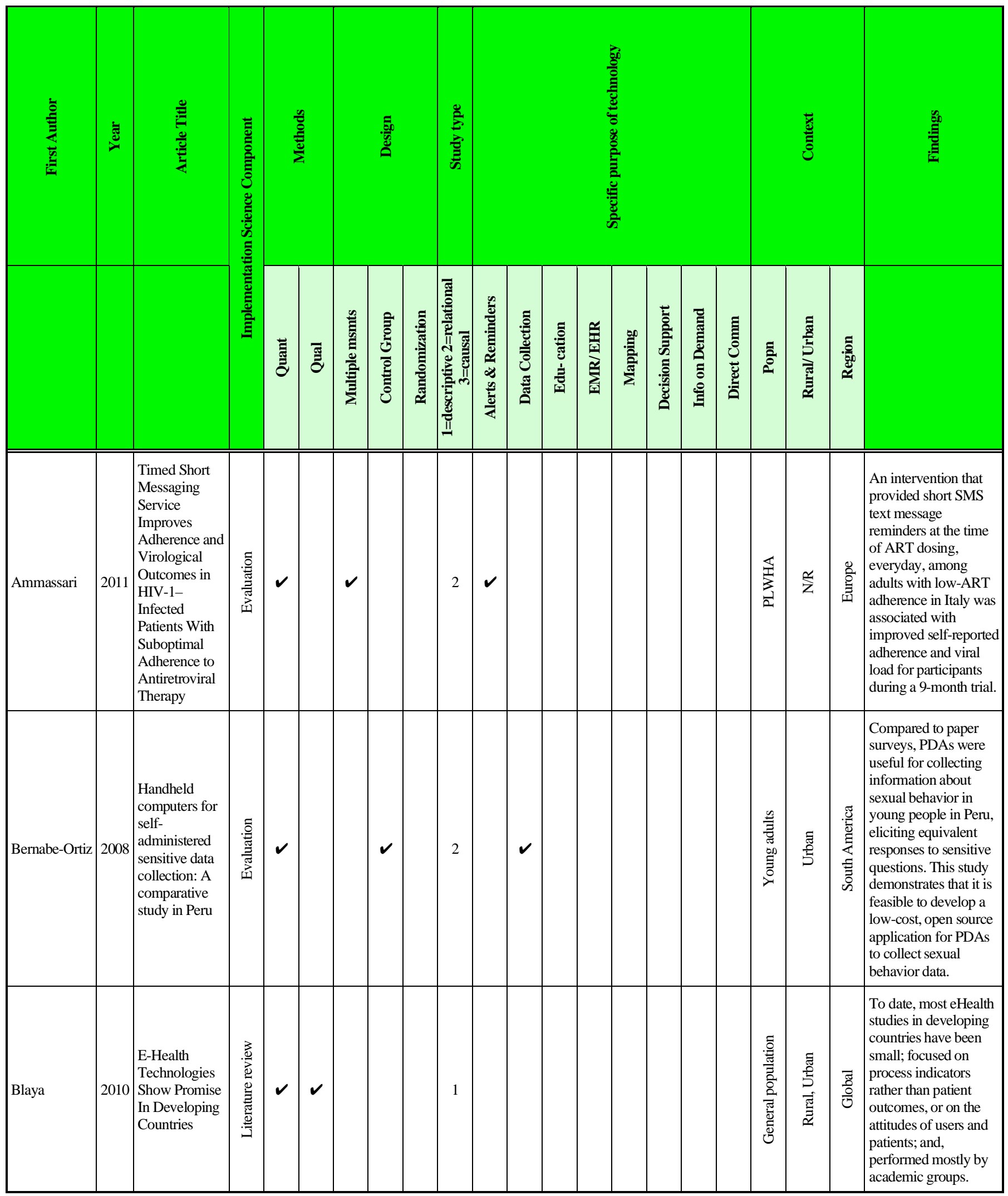


(Table 2) contd....

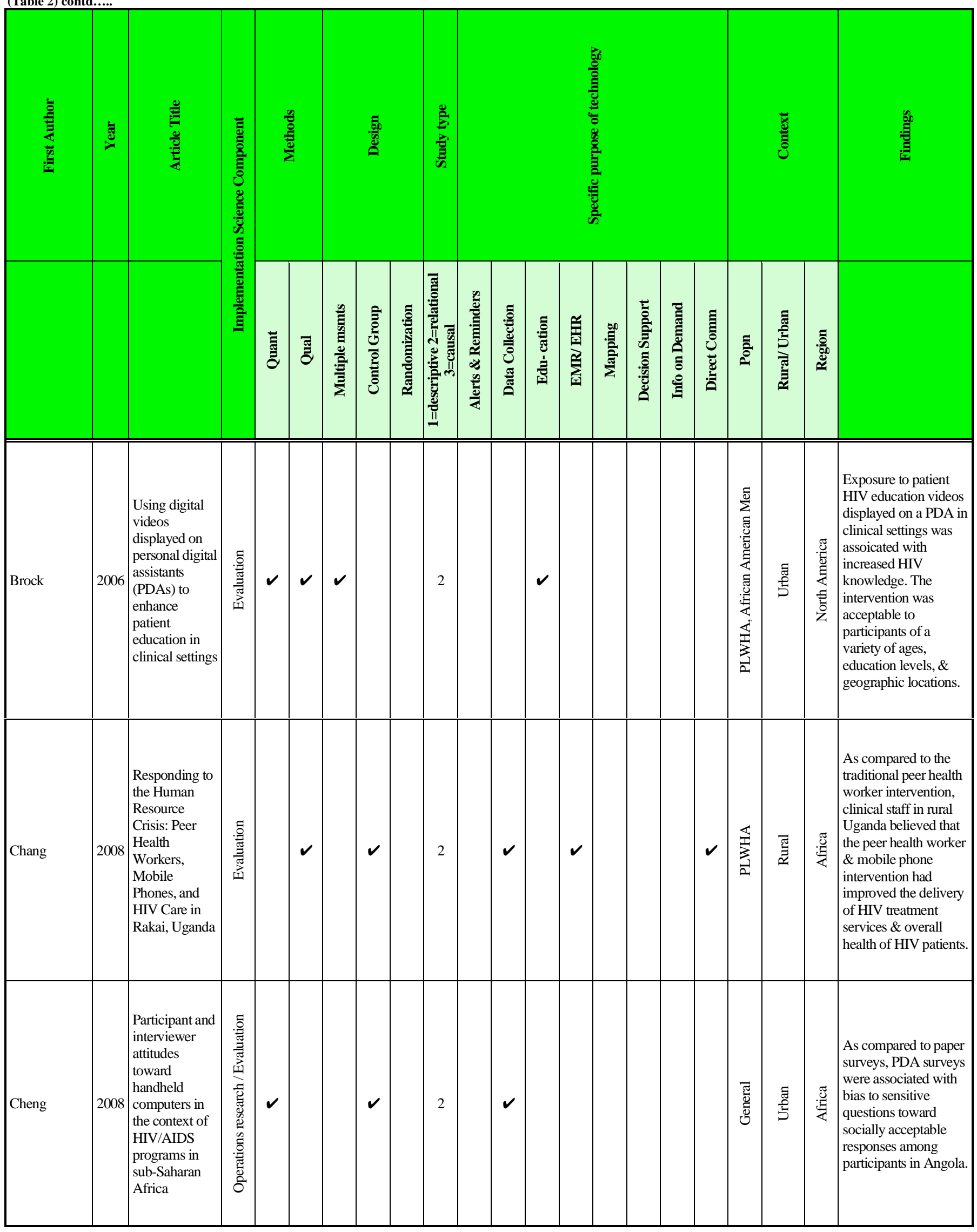




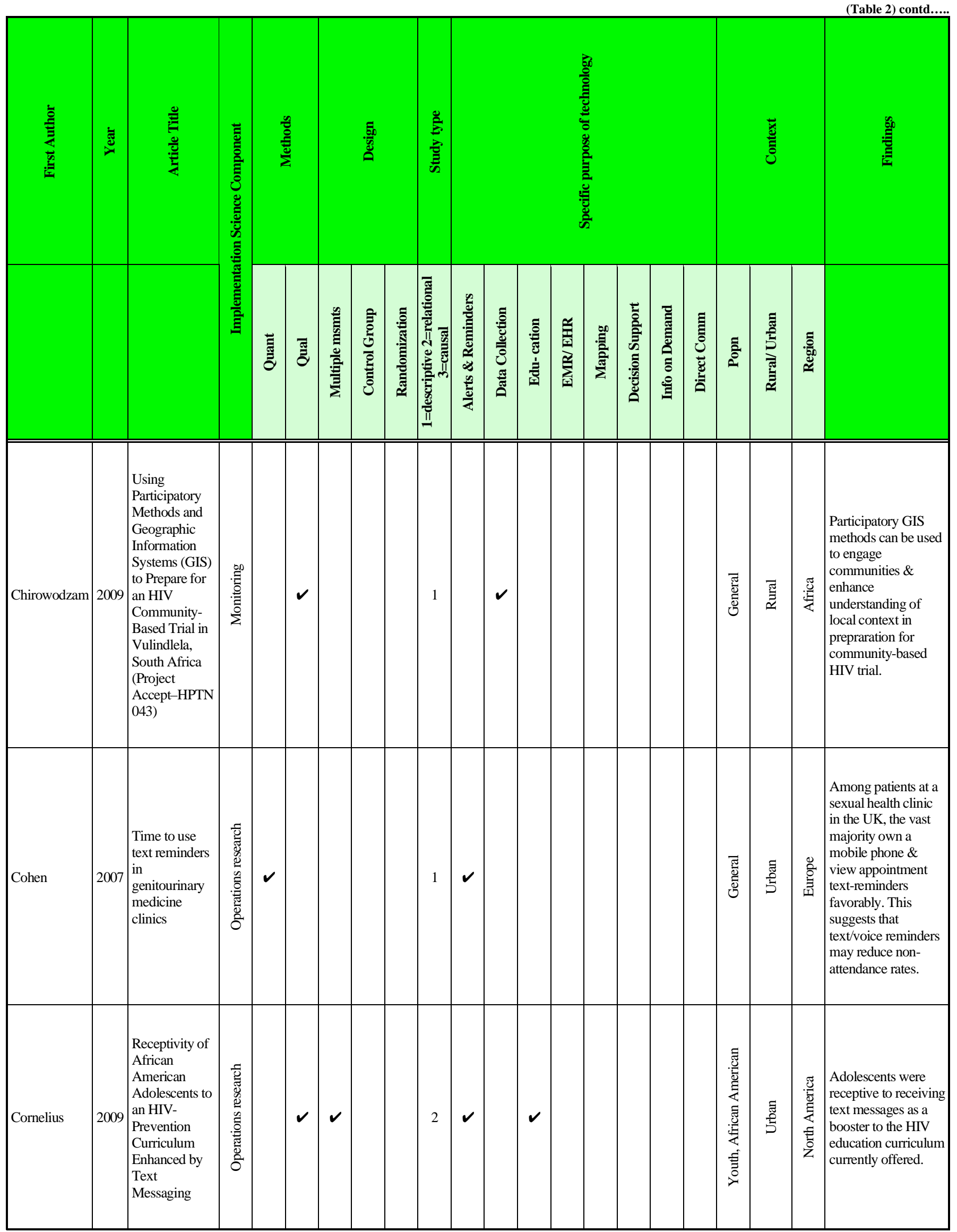


(Table 2) contd....

\begin{tabular}{|c|c|c|c|c|c|c|c|c|c|c|c|c|c|c|c|c|c|c|c|c|c|}
\hline \multirow[t]{2}{*}{ 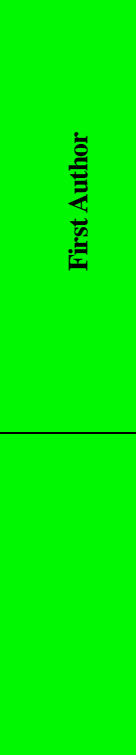 } & \multirow[t]{2}{*}{$\underset{\Xi}{\bar{\Xi}}$} & \multirow{2}{*}{\multicolumn{2}{|c|}{ 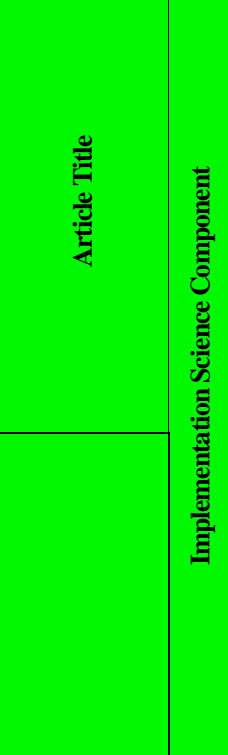 }} & \multicolumn{2}{|c|}{ 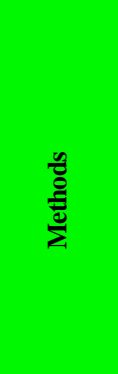 } & \multicolumn{3}{|c|}{ 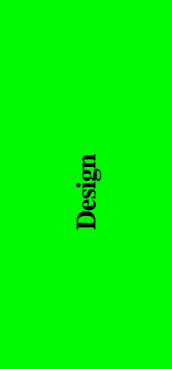 } & 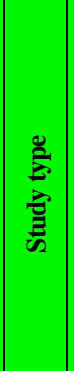 & \multicolumn{8}{|c|}{ 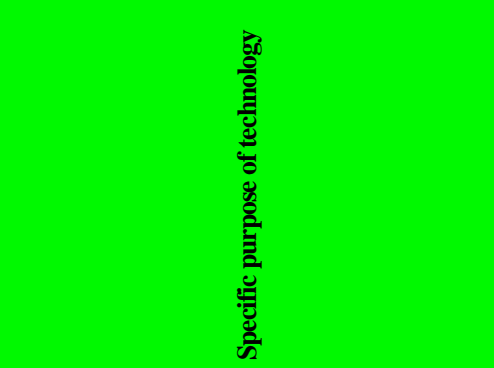 } & \multicolumn{3}{|c|}{$\begin{array}{l}\vec{E} \\
\vec{E}\end{array}$} & \multirow[t]{2}{*}{ 兽 } \\
\hline & & & & 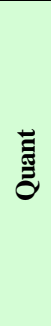 & $\overline{\tilde{\sigma}}$ & 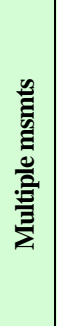 & 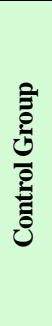 & 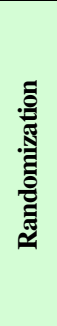 & 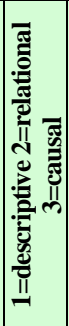 & 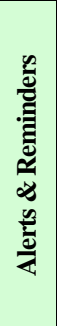 & 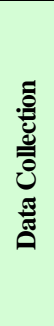 & 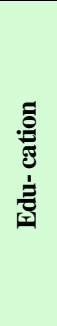 & 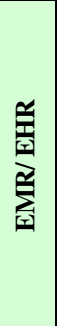 & & 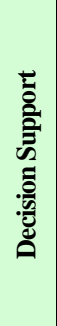 & 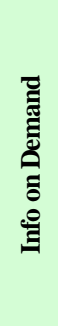 & 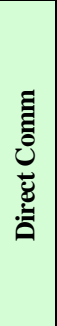 & ఏే & 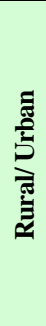 & 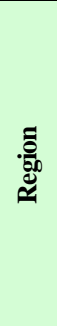 & \\
\hline Crankshaw & 2010 & $\begin{array}{l}\text { Exploring the } \\
\text { Patterns of Use } \\
\text { and the } \\
\text { Feasibility of } \\
\text { Using Cellular } \\
\text { Phones for } \\
\text { Clinic } \\
\text { Appointment } \\
\text { Reminders and } \\
\text { Adherence } \\
\text { Messages in an } \\
\text { Antiretroviral } \\
\text { Treatment } \\
\text { Clinic, Durban, } \\
\text { South Africa }\end{array}$ & 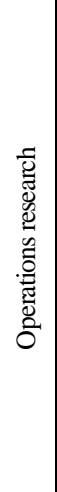 & $v$ & & & & & 1 & $\checkmark$ & & & & & & & & 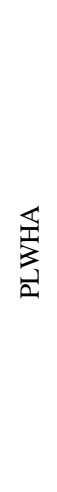 & 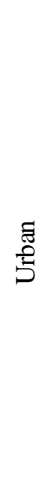 & $\underset{\mathbb{Z}}{\mathbb{Z}}$ & $\begin{array}{l}\text { The use of mobile } \\
\text { phones for HIV- } \\
\text { patient outreach \& } \\
\text { reminders is } \\
\text { acceptable to patients } \\
\text { \& feasible given } \\
\text { patient-reported } \\
\text { mobile phone use. } \\
\text { Interventions } \\
\text { targeting women } \\
\text { must consider higher } \\
\text { barriers to mobile } \\
\text { phone access. }\end{array}$ \\
\hline Curioso & 2005 & $\begin{array}{l}\text { Design and } \\
\text { Implementatio } \\
\text { n of Cell- } \\
\text { PREVEN: A } \\
\text { Real-Time } \\
\text { Surveillance } \\
\text { System for } \\
\text { Adverse } \\
\text { Events Using } \\
\text { Cell Phones in } \\
\text { Peru }\end{array}$ & 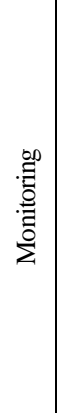 & $v$ & & & & & 1 & & $\boldsymbol{v}$ & & & & & & & $\frac{\frac{n}{0}}{\frac{0}{2}}$ & 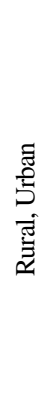 & 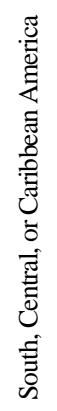 & $\begin{array}{l}\text { As demonstrated by } \\
\text { this pilot, it is } \\
\text { feasible to develop a } \\
\text { health surveillance } \\
\text { system based on } \\
\text { mobile phones for } \\
\text { real-time data } \\
\text { collection in Peru. }\end{array}$ \\
\hline Curioso & 2007 & $\begin{array}{l}\text { Access, use } \\
\text { and } \\
\text { perceptions } \\
\text { regarding } \\
\text { Internet, cell } \\
\text { phones and } \\
\text { PDAs as a } \\
\text { means for } \\
\text { health } \\
\text { promotion for } \\
\text { people living } \\
\text { with HIV in } \\
\text { Peru }\end{array}$ & 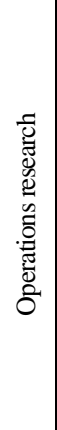 & & $\checkmark$ & & & & 1 & & & & & & & & & $\underset{3}{\mathbb{2}}$ & 趈 & 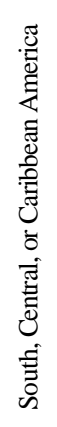 & $\begin{array}{l}\text { Health promotion } \\
\text { interventions using } \\
\text { information and } \\
\text { communication } \\
\text { technology tools } \\
\text { among people living } \\
\text { with HIV in } \\
\text { resource-constrained } \\
\text { settings may be } \\
\text { acceptable and } \\
\text { feasible, and can } \\
\text { build on existing } \\
\text { patterns of use. }\end{array}$ \\
\hline
\end{tabular}




\begin{tabular}{|c|c|c|c|c|c|c|c|c|c|c|c|c|c|c|c|c|c|c|c|c|c|}
\hline \multirow[t]{2}{*}{$\underbrace{}_{\bar{E}}$} & \multirow[t]{2}{*}{$\stackrel{\bar{\nexists}}{\bar{\nexists}}$} & \multirow[t]{2}{*}{ 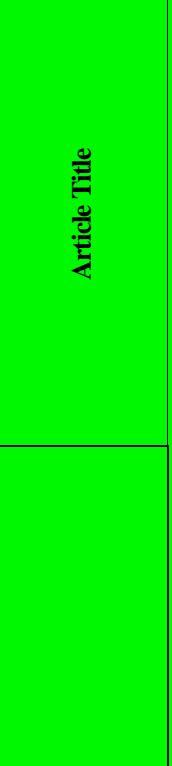 } & \multirow{2}{*}{ 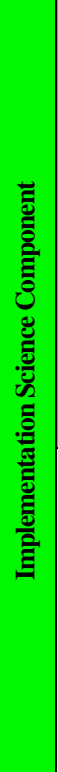 } & \multicolumn{2}{|c|}{ 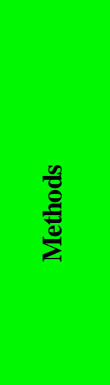 } & \multicolumn{3}{|c|}{ 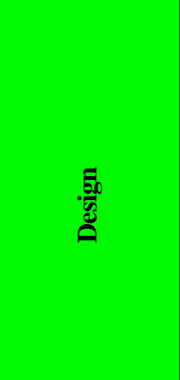 } & 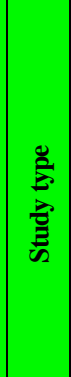 & \multicolumn{8}{|c|}{ 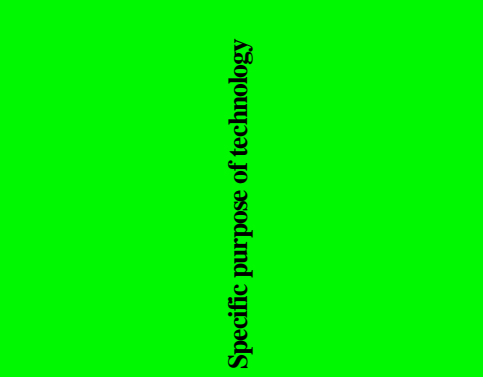 } & \multicolumn{3}{|c|}{$\begin{array}{l}\overrightarrow{0} \\
\overrightarrow{\tilde{e}} \\
\tilde{e}\end{array}$} & \multirow[t]{2}{*}{ 兽 } \\
\hline & & & & $\begin{array}{l}\vec{E} \\
\overline{\tilde{z}}\end{array}$ & $\overline{\tilde{\sigma}}$ & 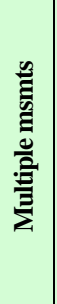 & 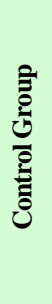 & 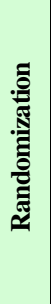 & 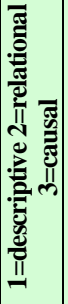 & 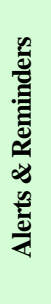 & 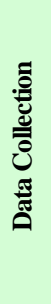 & 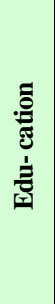 & 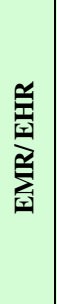 & $\frac{90}{\overline{\bar{n}}}$ & 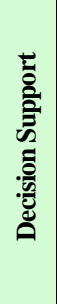 & 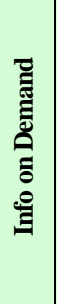 & 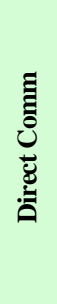 & $\stackrel{\Xi}{\varrho}$ & 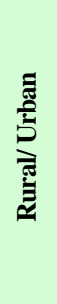 & 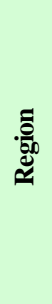 & \\
\hline Donald & 2007 & \begin{tabular}{|l|} 
Evaluation of \\
use of \\
cellphones to \\
aid compliance \\
with drug \\
therapy for \\
HIV patients
\end{tabular} & 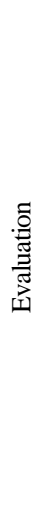 & & $\boldsymbol{v}$ & $\boldsymbol{V}$ & & & 2 & & $v$ & & & & & & $v$ & 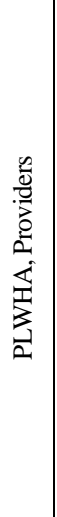 & 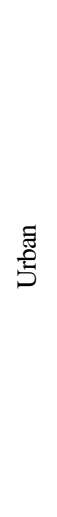 & 夏 & $\begin{array}{l}\text { Community health } \\
\text { workers found the } \\
\text { Cell-Life mobile } \\
\text { phone application to } \\
\text { be highly usable for } \\
\text { data collection, with } \\
\text { ease-of-use } \\
\text { improving during a } \\
\text { six-month period of } \\
\text { time. This suggests } \\
\text { that Cell-Life } \\
\text { facilitates the } \\
\text { management of } \\
\text { ARTs among } \\
\text { PLHIV in South } \\
\text { Africa. }\end{array}$ \\
\hline Dwolatzky & 2006 & \begin{tabular}{|l|} 
Linking the \\
global \\
positioning \\
system (GPS) \\
to a personal \\
digital assistant \\
(PDA) to \\
support \\
tuberculosis \\
control in \\
South Africa: a \\
pilot study
\end{tabular} & 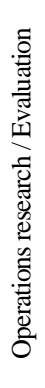 & $\checkmark$ & & & $\checkmark$ & & 2 & & & & & $\boldsymbol{v}$ & & & & 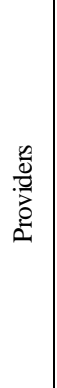 & 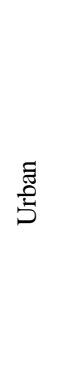 & 飬 & $\begin{array}{l}\text { GPS technology can } \\
\text { improve the ability to } \\
\text { locate and timeliness } \\
\text { in locating patients' } \\
\text { homes in informal } \\
\text { settlements in South } \\
\text { Africa. This suggests } \\
\text { that GPS may } \\
\text { improve the } \\
\text { efficiency of } \\
\text { HIV/TB treatment } \\
\text { adherence programs. }\end{array}$ \\
\hline Fraser & 2007 & \begin{tabular}{|l|} 
Information \\
Systems for \\
Patient Follow- \\
up and Chronic \\
Management \\
of HIV and \\
Tuberculosis: a \\
Life-saving \\
Technology in \\
Resource-poor \\
Areas
\end{tabular} & 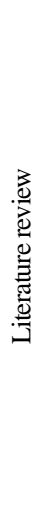 & & $v$ & & & & 1 & $\checkmark$ & $\checkmark$ & & $\boldsymbol{v}$ & & & & & 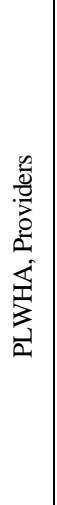 & 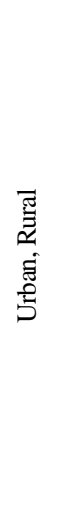 & $\begin{array}{l}\text { न्ञ } \\
\text { 음 }\end{array}$ & $\begin{array}{l}\text { Projects demonstrate } \\
\text { that information } \\
\text { systems can support } \\
\text { and monitor HIV } \\
\text { and MDR-TB } \\
\text { projects as they scale } \\
\text { up, tracking patients } \\
\text { from initial diagnosis } \\
\text { through treatment } \\
\text { adherence. However, } \\
\text { there is a lack of } \\
\text { quantitative \& } \\
\text { impact evaluations in } \\
\text { the literature to } \\
\text { scientifically confirm } \\
\text { this suggestion. }\end{array}$ \\
\hline
\end{tabular}




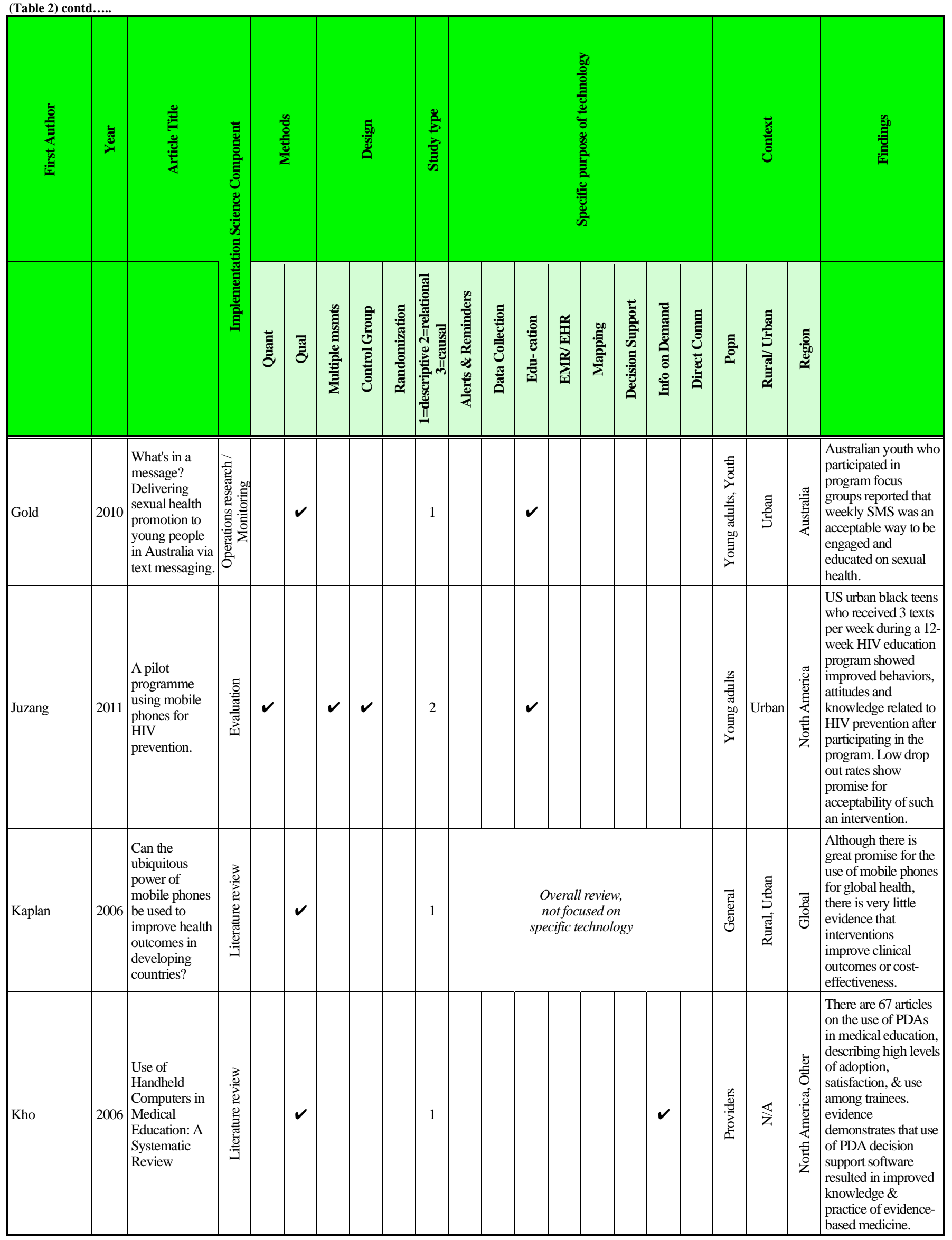




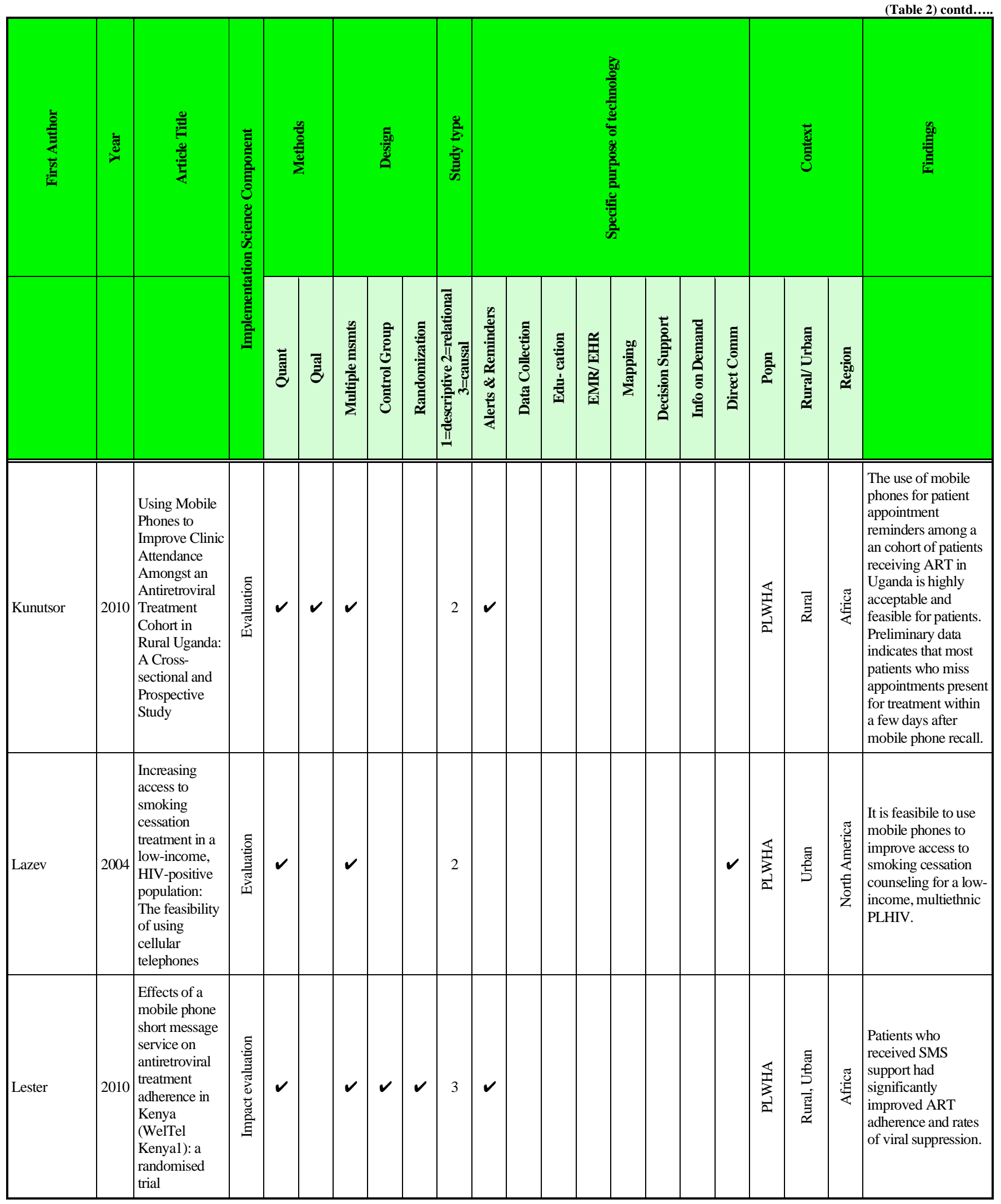




\begin{tabular}{|c|c|c|c|c|c|c|c|c|c|c|c|c|c|c|c|c|c|c|c|c|c|}
\hline \multirow[t]{2}{*}{ 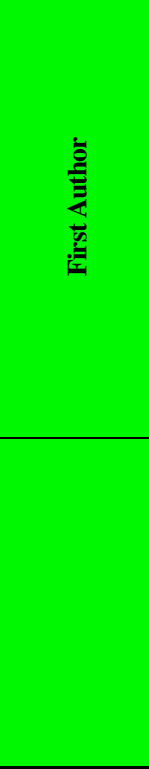 } & \multirow[t]{2}{*}{$\stackrel{\bar{\Xi}}{\grave{\Xi}}$} & \multirow{2}{*}{\multicolumn{2}{|c|}{ 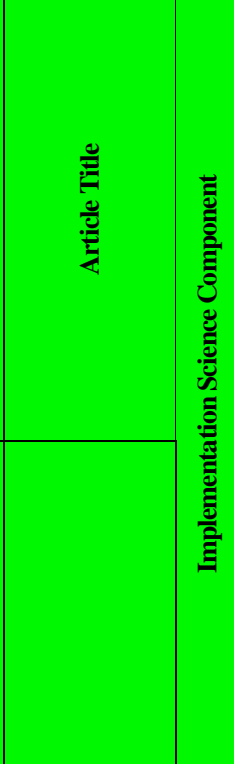 }} & \multicolumn{2}{|c|}{ 莺 } & \multicolumn{3}{|c|}{ 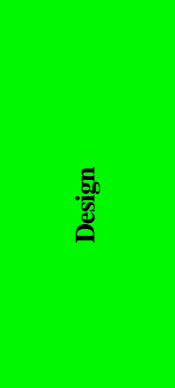 } & \multirow{2}{*}{ 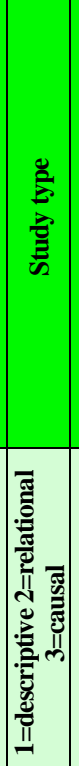 } & \multicolumn{8}{|c|}{ 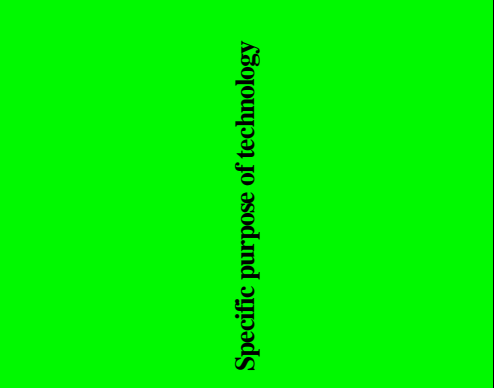 } & \multicolumn{3}{|c|}{ U⿺尢丶 } & \multirow[t]{2}{*}{ 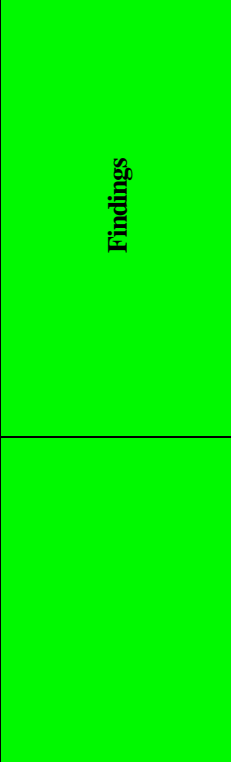 } \\
\hline & & & & 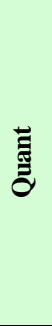 & $\overline{\tilde{\sigma}}$ & 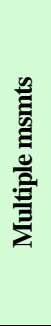 & 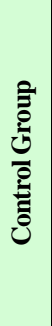 & 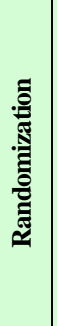 & & 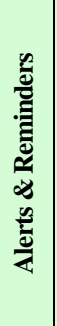 & 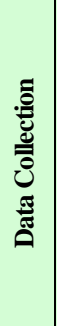 & 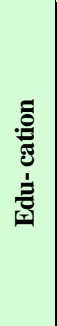 & 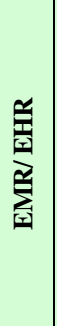 & 最 & 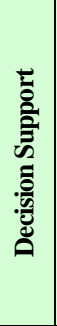 & 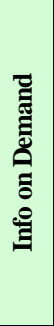 & 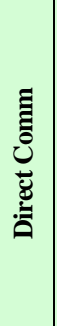 & aี & 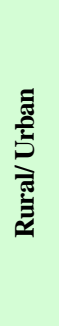 & 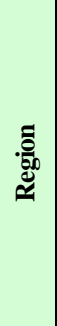 & \\
\hline Levine & 2008 & $\begin{array}{l}\text { SEXINFO: a } \\
\text { Sexual Health } \\
\text { Text } \\
\text { Messaging } \\
\text { Service for San } \\
\text { Francisco } \\
\text { Youth }\end{array}$ & 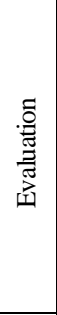 & $\checkmark$ & & & & & 1 & & & & & & & $\boldsymbol{V}$ & & $\stackrel{\Xi}{\Xi}$ & 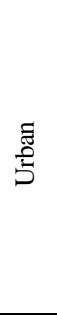 & 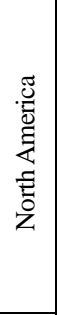 & $\begin{array}{l}\text { SexInfo SMS referral } \\
\text { service is a feasible } \\
\text { and acceptable way } \\
\text { for at-risk youths in } \\
\text { San Francisco to } \\
\text { receive sexual health } \\
\text { information and } \\
\text { referrals to sexual } \\
\text { and reproductive } \\
\text { health services. }\end{array}$ \\
\hline Perron & 2010 & $\begin{array}{l}\text { Reduction of } \\
\text { missed } \\
\text { appointments } \\
\text { at an urban } \\
\text { primary care } \\
\text { clinic: a } \\
\text { randomised } \\
\text { controlled } \\
\text { study }\end{array}$ & 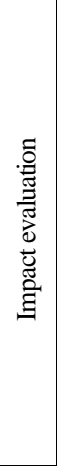 & $\checkmark$ & & & $\checkmark$ & $\checkmark$ & 3 & $\boldsymbol{V}$ & & & & & & & & 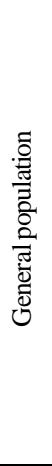 & $\begin{array}{l}\text { : } \\
\text { Dేّ }\end{array}$ & 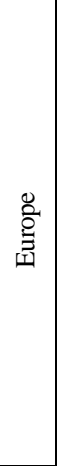 & $\begin{array}{l}\text { A patient reminder } \\
\text { system, including } \\
\text { SMS reminders, } \\
\text { significantly } \\
\text { increased patient } \\
\text { attendance at } \\
\text { medical outpatient } \\
\text { clinics in Geneva. } \\
\text { An intervention } \\
\text { focused on specific } \\
\text { patient characteristics } \\
\text { may further increase } \\
\text { the effectiveness of } \\
\text { appointment } \\
\text { reminders. }\end{array}$ \\
\hline Pop-Eleches & 2010 & \begin{tabular}{|l|} 
Mobile phone \\
technologies \\
improve \\
adherence to \\
antiretroviral \\
treatment in a \\
resource- \\
limited setting: \\
a randomized \\
controlled trial \\
of text message \\
reminders
\end{tabular} & 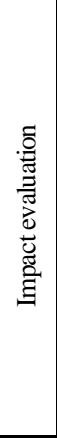 & $\checkmark$ & & $\checkmark$ & $\checkmark$ & $\checkmark$ & 3 & $\boldsymbol{v}$ & & & & & & & & 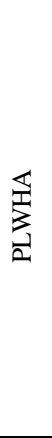 & 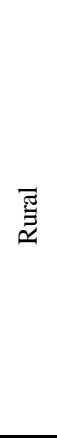 & $\underset{⿱ 乛 龰}{\mathbb{2}}$ & $\begin{array}{l}\text { These results suggest } \\
\text { that SMS reminders } \\
\text { may be an important } \\
\text { tool to achieve } \\
\text { optimal treatment } \\
\text { response in resource- } \\
\text { limited settings. } \\
\text { Overall, weekly text } \\
\text { message reminders } \\
\text { increase adherence to } \\
\text { ART. Daily } \\
\text { reminders, on the } \\
\text { other hand, failed to } \\
\text { increase adherence. }\end{array}$ \\
\hline
\end{tabular}




\begin{tabular}{|c|c|c|c|c|c|c|c|c|c|c|c|c|c|c|c|c|c|c|c|c|c|}
\hline \multirow[t]{2}{*}{ 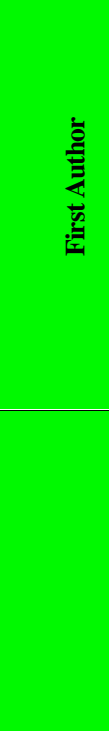 } & \multirow[t]{2}{*}{ ટ્ } & \multirow{2}{*}{\multicolumn{2}{|c|}{ 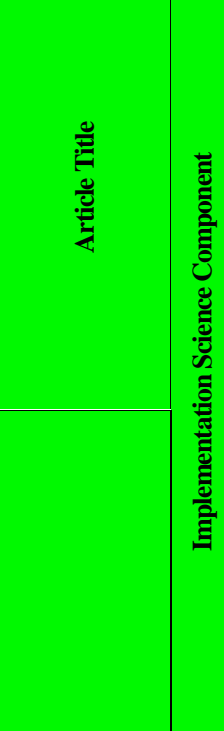 }} & \multicolumn{2}{|c|}{ 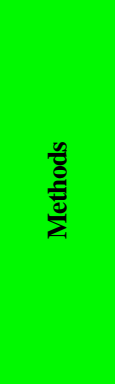 } & \multicolumn{4}{|c|}{$\begin{array}{l}\frac{\vec{g}}{\bar{g}} \\
\stackrel{\bar{g}}{0}\end{array}$} & \multicolumn{8}{|c|}{ 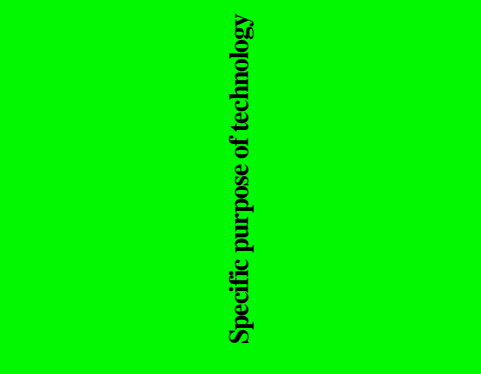 } & \multicolumn{3}{|c|}{$\begin{array}{l}\overrightarrow{0} \\
\vec{E}\end{array}$} & \multirow[t]{2}{*}{ 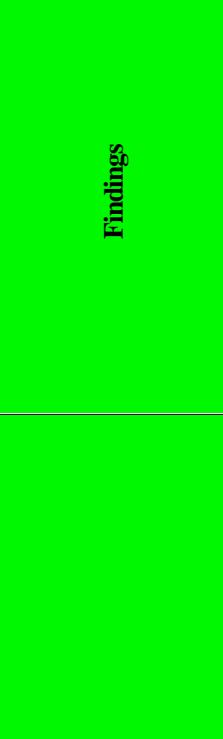 } \\
\hline & & & & $\overline{\overline{\bar{z}}}$ & $\overline{\mathrm{J}}$ & 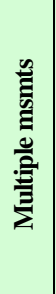 & 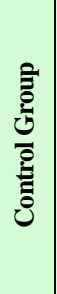 & . & 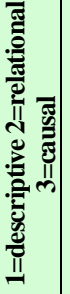 & 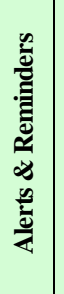 & 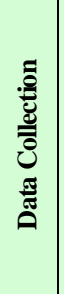 & 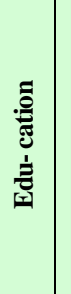 & $\sum_{\text {竞 }}^{\text {竞 }}$ & 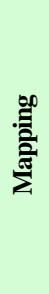 & 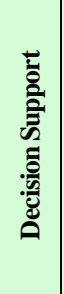 & 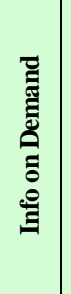 & 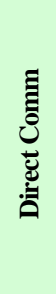 & $\stackrel{\bar{a}}{\varrho}$ & 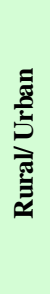 & 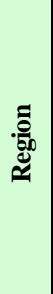 & \\
\hline Puccio & 2006 & \begin{tabular}{|l||} 
The Use of \\
Cell Phone \\
Reminder \\
Calls for \\
Assisting HIV- \\
Infected \\
Adolescents \\
and Young \\
Adults to \\
Adhere to \\
Highly Active \\
Antiretroviral \\
Therapy: A \\
Pilot Study \\
\end{tabular} & 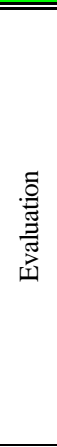 & $v$ & $v$ & $v$ & & & 2 & $v$ & & & & & & & $v$ & 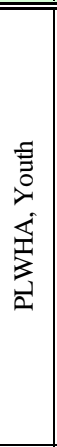 & $\begin{array}{l}\text { ह } \\
\text { క్ }\end{array}$ & 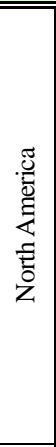 & $\begin{array}{l}\text { Mobile phone } \\
\text { reminder calls were } \\
\text { acceptible \& } \\
\text { feasible. There is } \\
\text { some indication that } \\
\text { reminders contribute } \\
\text { to ART adherence, } \\
\text { however, viral } \\
\text { supression at the } \\
\text { close of the 12-week } \\
\text { study was } \\
\text { inadequate. }\end{array}$ \\
\hline Samal & 2010 & \begin{tabular}{|l|} 
Digital Divide: \\
Variation in \\
Internet and \\
Cellular Phone \\
Use Among \\
Women \\
Attending an \\
Urban \\
Sexually \\
Transmitted \\
Infections \\
Clinic
\end{tabular} & 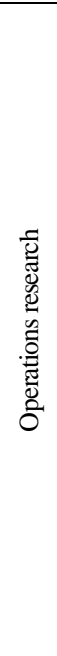 & $v$ & & & & & 1 & & & & $\begin{array}{l}\text { ot foct } \\
\text { cificte }\end{array}$ & chno & & & & $\begin{array}{l}\overline{\bar{v}} \\
\text { हैं }\end{array}$ & 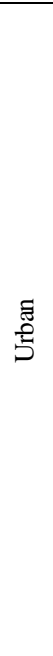 & 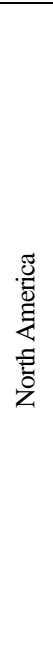 & $\begin{array}{l}\text { Although the } \\
\text { majority of } \\
\text { Baltimore patient } \\
\text { respondents found } \\
\text { ICTs acceptible, use } \\
\text { of computers and } \\
\text { internet was related } \\
\text { to being young and } \\
\text { educated while } \\
\text { mobile phones use } \\
\text { was more equitable. } \\
\text { Web-based } \\
\text { interventions for } \\
\text { urban women may } \\
\text { serve to widen health } \\
\text { disparities, however } \\
\text { mobile phone } \\
\text { interventions may be } \\
\text { more appropriate } \\
\text { among underserved } \\
\text { communities. }\end{array}$ \\
\hline Shacham & 2009 & \begin{tabular}{|l|} 
Can you hear \\
me now? \\
Limited use of \\
technology \\
among an \\
urban HIV- \\
infected cohort
\end{tabular} & 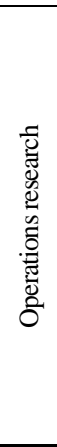 & $\checkmark$ & & & & & 1 & & & & cifict & chnol & & & & 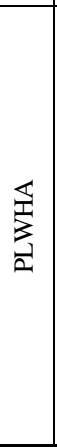 & $\begin{array}{l}\overline{0} \\
\text { है }\end{array}$ & 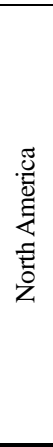 & $\begin{array}{l}\text { Disparities among } \\
\text { internet, cell phone, } \\
\text { and text messaging } \\
\text { usage exist among } \\
\text { HIV-infected } \\
\text { individuals. In this } \\
\text { particular urban US } \\
\text { cohort of PLHIV, } \\
\text { cell phone ownership } \\
\text { was associated with } \\
\text { being Caucasian, } \\
\text { employed, more } \\
\text { educated, and higher } \\
\text { salary. }\end{array}$ \\
\hline
\end{tabular}


(Table 2) contd.....

\begin{tabular}{|c|c|c|c|c|c|c|c|c|c|c|c|c|c|c|c|c|c|c|c|c|c|}
\hline \multirow[t]{2}{*}{ 离 } & \multirow[t]{2}{*}{$\underset{\bar{\Xi}}{\grave{\Xi}}$} & \multirow{2}{*}{\multicolumn{2}{|c|}{ 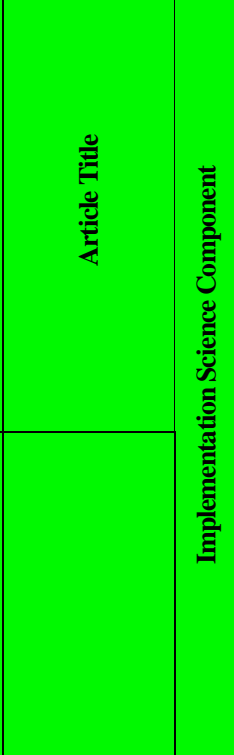 }} & \multicolumn{2}{|c|}{$\begin{array}{l}\frac{n}{8} \\
\frac{E}{\tilde{E}} \\
\Sigma\end{array}$} & \multicolumn{3}{|c|}{ 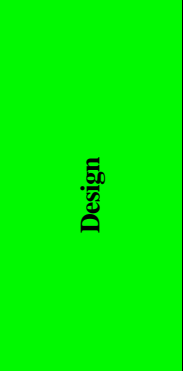 } & 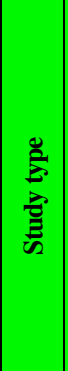 & \multicolumn{8}{|c|}{ 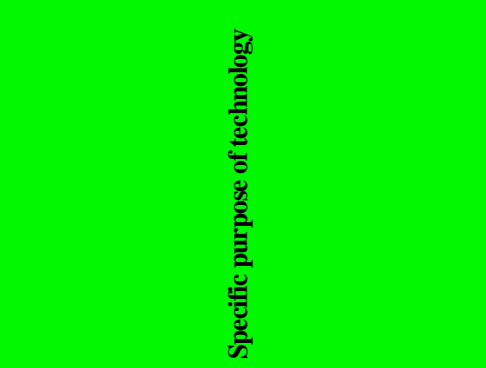 } & \multicolumn{3}{|c|}{$\begin{array}{l}\overrightarrow{\mathrm{x}} \\
\overrightarrow{\mathrm{z}}\end{array}$} & \multirow[t]{2}{*}{ 总 } \\
\hline & & & & $\begin{array}{l}\overrightarrow{\bar{E}} \\
\bar{\Xi}\end{array}$ & $\overline{\tilde{\sigma}}$ & 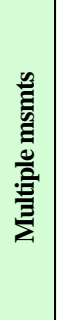 & 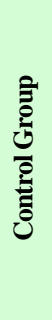 & 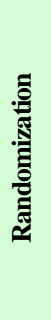 & 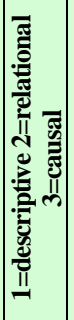 & 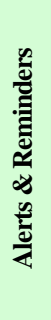 & 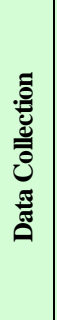 & 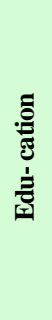 & 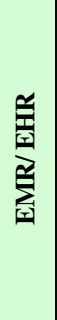 & 最 & 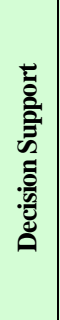 & 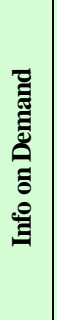 & 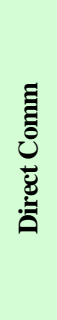 & పี & 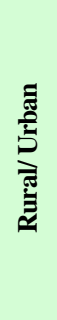 & 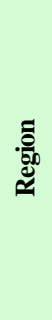 & \\
\hline Shet & 2010 & \begin{tabular}{|l|} 
Designing a \\
Mobile Phone- \\
Based \\
Intervention to \\
Promote \\
Adherence to \\
Antiretroviral \\
Therapy in \\
South India
\end{tabular} & 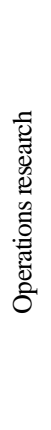 & $\boldsymbol{V}$ & & & & & 1 & $\checkmark$ & & & & & & & & 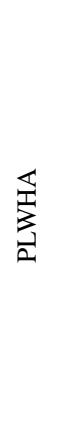 & 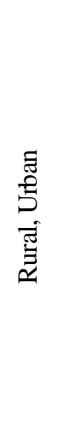 & 吾 & $\begin{array}{l}\text { Patient attitudes } \\
\text { about and access to } \\
\text { mobile phones } \\
\text { indicates that } \\
\text { delivering ART } \\
\text { adherence services, } \\
\text { such as weekly } \\
\text { automated voice } \\
\text { reminders, via } \\
\text { mobile phone in } \\
\text { South India is } \\
\text { feasible and } \\
\text { acceptable. }\end{array}$ \\
\hline Vidrine & 2006 & $\begin{array}{l}\text { A randomized } \\
\text { trial of a } \\
\text { proactive } \\
\text { cellular } \\
\text { telephone } \\
\text { intervention for } \\
\text { smokers living } \\
\text { with } \\
\text { HIV/AIDS }\end{array}$ & 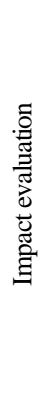 & $\checkmark$ & & $\boldsymbol{v}$ & $\checkmark$ & $\checkmark$ & 3 & & & & & & & & $\checkmark$ & 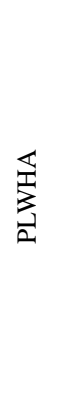 & $\begin{array}{l}\text { : } \\
\text { :ैं }\end{array}$ & 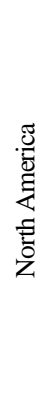 & $\begin{array}{l}\text { As opposed to the } \\
\text { usual care approach, } \\
\text { those PLHIV who } \\
\text { participated in a } \\
\text { smoking cessation } \\
\text { program with } 8 \\
\text { mobile phone } \\
\text { counseling sessions } \\
\text { were } 3.6 \text { times more } \\
\text { likely to have quit } \\
\text { smoking at } 3- \\
\text { months. }\end{array}$ \\
\hline Vidrine & 2006 & \begin{tabular}{|l|} 
Impact of a \\
Cell Phone \\
Intervention on \\
Mediating \\
Mechanisms of \\
Smoking \\
Cessation in \\
Individuals \\
Living with \\
HIV/AIDS
\end{tabular} & 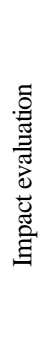 & $\boldsymbol{V}$ & & $\boldsymbol{V}$ & $\checkmark$ & $\checkmark$ & 3 & & & & & & & & $\checkmark$ & 焉 & 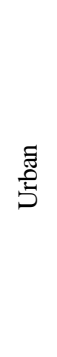 & 后 & $\begin{array}{l}\text { See above. } \\
\text { Additionally, } \\
\text { participants in the } \\
\text { mobile phone } \\
\text { counseling } \\
\text { intervention } \\
\text { experienced greater } \\
\text { reductions in anxiety } \\
\text { and depression and } \\
\text { increases in self- } \\
\text { effiicacy. }\end{array}$ \\
\hline
\end{tabular}




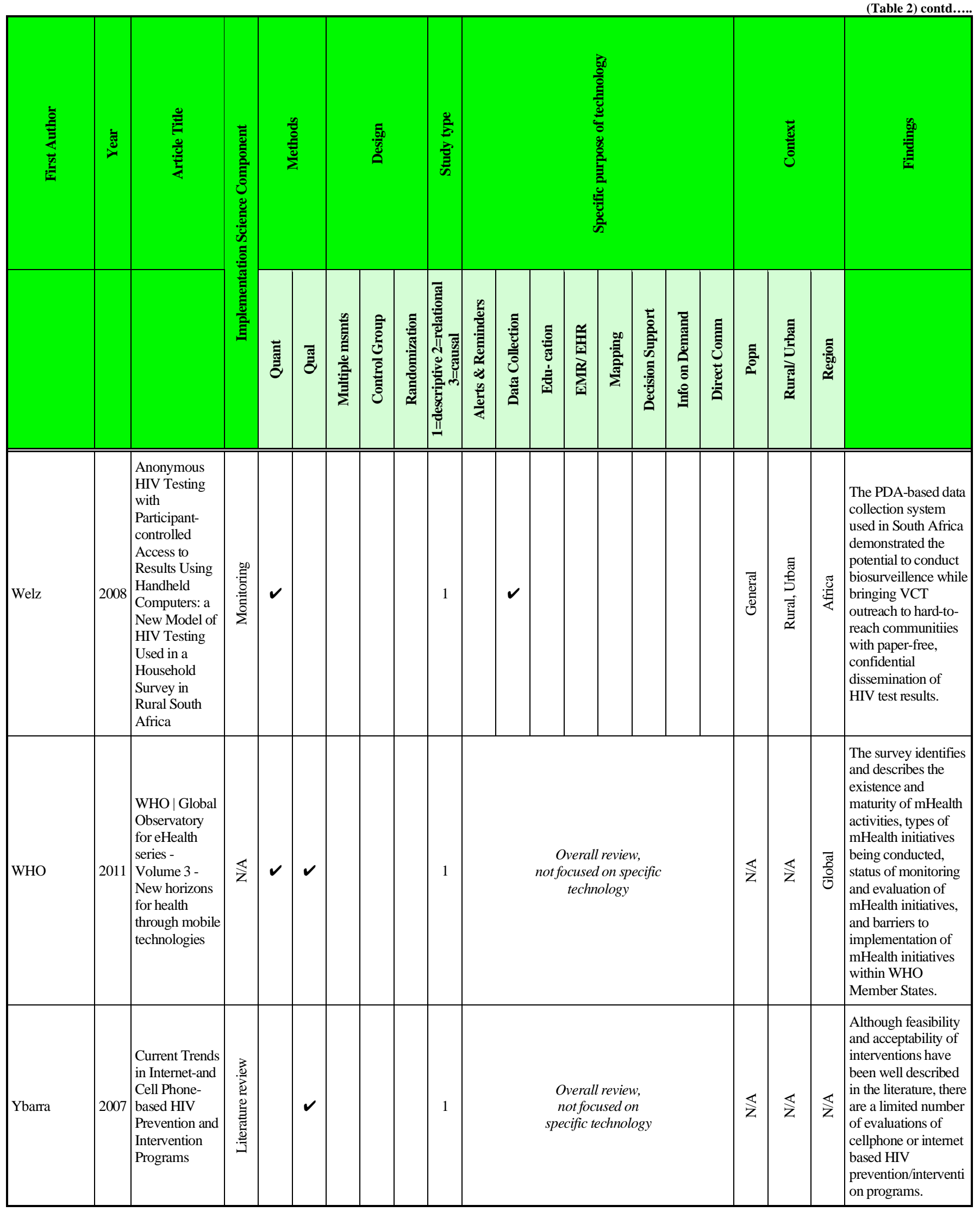


The literature was then divided into two categories: research and non-research. Research articles were defined as those that reported on systematic investigation of an mHealth implementation or tool using a specified study design and methods. The majority of articles were excluded from the research category because they had no description of methods used for investigation. These articles tended to describe an mHealth for HIV/AIDS implementation, often including lessons learned. Two articles were excluded because they described research that happened to use mHealth tools for the purpose of conducting a survey but not provide explicit research insights into the use of the mHealth tool itself. All articles were used in the assessment of the overall landscape of mHealth for HIV/AIDS and only research articles were used in the assessment of the evidence related to the use of mHealth to address specific HIV programmatic requirements.

\section{FINDINGS}

\section{mHealth \& HIV Landscape}

The mHealth literature collection included 2,603 articles, after sorting to include only unique articles that were in line with the above stated definition of mHealth. The majority of those articles considered irrelevant to mHealth described the unhealthy effects of mobile phones, such as exposure to electromagnetic signals and phone use while driving. Within the final mHealth collection, 64 were deemed pertinent to HIV/AIDS after the review of title, abstract, and author keywords. The team included additional articles from reference lists of included articles and then ensured relevance to mHealth and HIV with a full article review, resulting in a final collection of 62 articles for analysis. These detailed are depicted in Fig. (1): PRISMA flow diagram.

As described in the literature review tables (Tables $\mathbf{1}$ and 2) and overview table (Table 3), the final collection of 62 articles were mostly from peer-reviewed journals and included policy and position papers, editorial commentary, literature reviews, and research/evaluation. Articles published from 2001-2006 tended to focus on the use of personal digital assistants (PDAs) to support provider services. Nearly $80 \%$ of all articles were published between 2007 and 2011, focusing more on the use of mobile phones to support providers, community health workers, and patients directly. Among the ten literature reviews found, only two were systematic and included a description of methods $[15,16]$. These reviews were tangentially related to HIV/AIDS, tending to focus more broadly on mobile devices for medical education or health services in developing countries.

Although articles describe using a range of technical capabilities, most focus on simple text-based SMS. More than half of projects provided alerts and reminders $(60 \%)$ and a quarter used mobile tools for field-based data collection (24\%) such as surveys and electronic patient diaries. A smaller number of studies described the use of mobile technology for direct voice communication between patients and providers (24\%), HIV information on demand (11\%), educational messaging and health promotion $(13 \%)$, and HIV provider decision-support (5\%). Only one article, a brief news report, described the use of mobile technology for point-of-care diagnostics, specifically a mobile phone imaging tool that is currently in development to measure CD4 count from remote locations [17].

Table 3. Overview of mHealth Literature

\begin{tabular}{|c|c|c|c|}
\hline Category & Subcategory & $\begin{array}{c}\text { Result } \\
\text { (n) }\end{array}$ & $\begin{array}{c}\text { Result } \\
(\%)\end{array}$ \\
\hline \multirow{2}{*}{ Research } & Yes & 35 & $56 \%$ \\
\hline & No & 27 & $44 \%$ \\
\hline \multirow{2}{*}{ Peer-reviewed } & Yes & 45 & $73 \%$ \\
\hline & No & 13 & $21 \%$ \\
\hline \multirow{2}{*}{ Rural/ Urban } & Urban & 44 & $71 \%$ \\
\hline & Rural & 28 & $45 \%$ \\
\hline \multirow{10}{*}{$\begin{array}{c}\text { Specific } \\
\text { intervention } \\
\text { population }\end{array}$} & PLWHA & 28 & $45 \%$ \\
\hline & General Popn & 17 & $27 \%$ \\
\hline & Providers & 7 & $11 \%$ \\
\hline & Youth/Teens/Young adults & 6 & $10 \%$ \\
\hline & Women & 2 & $3 \%$ \\
\hline & Pregnant Women & 2 & $3 \%$ \\
\hline & Infants/Children & 1 & $2 \%$ \\
\hline & Sex workers & 3 & $5 \%$ \\
\hline & Men who have sex w men & 3 & $5 \%$ \\
\hline & Users of injection drugs & 0 & $0 \%$ \\
\hline \multirow{5}{*}{ Region } & Africa & 18 & $29 \%$ \\
\hline & North America & 14 & $23 \%$ \\
\hline & Europe & 5 & $8 \%$ \\
\hline & Latin America & 4 & $6 \%$ \\
\hline & Asia & 3 & $5 \%$ \\
\hline \multirow{8}{*}{$\begin{array}{l}\text { Purpose of } \\
\text { technology }\end{array}$} & Alerts \& reminders & 37 & $60 \%$ \\
\hline & Data collection & 15 & $24 \%$ \\
\hline & Direct voice communication & 15 & $24 \%$ \\
\hline & Educational messaging & 8 & $13 \%$ \\
\hline & Info on Demand & 7 & $11 \%$ \\
\hline & EMR/EHR & 4 & $6 \%$ \\
\hline & Decision Support & 3 & $5 \%$ \\
\hline & Mapping & 2 & $3 \%$ \\
\hline
\end{tabular}

The literature describes a proliferation of mHealth tools for HIV across geographic regions and within diverse communities of focus. The majority of projects provided services to urban communities in the global South. Projects mostly focused on providing direct services to people living with HIV (PLHIV) (45\%). Only a few articles mentioned a focus on key vulnerable populations, such as pregnant women $(3 \%)$, sex workers $(5 \%)$, men who have sex with men $(5 \%)$, and users of injection drugs $(0 \%)$. 


\section{Research \& Evaluation Studies}

Of 62 articles identified, $56 \%$ were research or evaluation studies -- ie, they focused on systematic investigation of mHealth for HIV and reported using a specific study design and methods. As detailed in Table 4, these studies can be generally characterized by the fundamental components of implementation science: monitoring/evaluation, operations research, and impact evaluation.

\section{Table 4. Overview of mHealth Research}

\begin{tabular}{|c|c|c|c|}
\hline \multirow{3}{*}{ Category } & Subcategory & Result (n) & Result (\%) \\
\hline \hline \multirow{3}{*}{$\begin{array}{c}\text { Implementation } \\
\text { Science Category }\end{array}$} & $\begin{array}{c}\text { Monitoring/ } \\
\text { evaluation reports }\end{array}$ & 16 & $17 \%$ \\
\cline { 2 - 4 } & Operations research & 10 & $46 \%$ \\
\cline { 2 - 4 } & Impact evaluation & 5 & $14 \%$ \\
\hline \multirow{3}{*}{ Methods } & Quantitative & 24 & $69 \%$ \\
\cline { 2 - 4 } & Qualitative & 16 & $46 \%$ \\
\hline \multirow{2}{*}{ Design } & Multiple measurements & 12 & $34 \%$ \\
\cline { 2 - 4 } & Control group & 10 & $29 \%$ \\
\cline { 2 - 4 } & Randomization & 5 & $14 \%$ \\
\hline
\end{tabular}

Most studies can be characterized as monitoring/evaluation reports. Four studies (20\%) were monitoring reports, particularly descriptions of project activities, inputs, and outputs. A third of studies (34\%) were evaluation reports, including relational/quasi-experimental studies of what was achieved by mHealth initiatives. Monitoring and evaluation efforts of this kind often included taking multiple measurements of programs or interventions across time, such as pre/post tests. Typical of this kind of study, Brock and Smith assessed the use of a mobile device with education videos in a clinic waiting room to measure patients' knowledge of HIV/AIDS before, immediately after, and 4-6 weeks after use [18]. The study found that participating patients had higher HIV/AIDS knowledge scores after using the mHealth educational tool, however it did not include control groups to compare the mHealth system in relation to no intervention or other recommended educational practices.

Ten studies $(29 \%)$ reported on operations research, including many feasibility and acceptability assessments that focus on how an mHealth solution might be most efficiently adopted within a particular clinical or community context to improve program practices. These articles did not involve a comparison group, multiple measurements, or randomization but more often described results of cross-sectional surveys, focus groups, and interviews with community stakeholder groups. For example, Crankshaw et al. evaluated the use of mobile phones for clinic appointment reminders and adherence messages at an antiretroviral treatment (ART) clinic in urban South Africa that was struggling with loss to follow-up [19]. Their findings indicated that although most patients had access to a mobile phone, women patients tended to change phones and share phones more often than men. The authors argue that these feasibility findings must be incorporated into program delivery to protect patient privacy and improve program effectiveness.

Finally, the literature collection included several impact evaluations (14\%) aimed to assess causality using experimental design with randomized selection of intervention and control groups. Efforts of this kind tended to focus on measuring program outcomes such as treatment adherence and biomedical health impacts such as CD4count. Randomized clinical studies (RCTs) led by PopEleches et al. and Lester et al. (findings described below) are salient examples of rigorous studies [20,21].

Overall, studies used a mix of quantitative (71\%) and qualitative $(51 \%)$ methods, including surveys, focus groups, clinical chart review, and computer systems reports on use. Although some (14\%) used both qualitative/quantitative strategies, none described an integrated mixed method approach. Among 35 studies, only a few [22-27] mentioned a theoretical basis or conceptual framework that guided their assumptions about why mHealth might facilitate or cause the intended change.

\section{HIV Programmatic Objectives}

The research literature provided evidence on a range of interventions, several of which might contribute evidence to the ways in which mHealth for HIV might facilitate the scale-up of HIV prevention, care, and treatment services across the cascade, as depicted in Fig. (2).

Voluntary counseling \& testing: Three articles provide some evidence that mHealth tools can be used to increase demand for voluntary counseling and testing (VCT) among vulnerable communities. First, in their assessment of the SEXINFO text messaging service in San Francisco, Levine and colleagues found that SMS was a feasible and acceptable way for vulnerable youth to receive information and referrals to clinical services [28]. Using systems data, the researchers were able to assess project outputs and found that the project resulted in a sizeable number of messages sent and received by youth. However, the study did not assess whether or not SEXINFO had an impact on VCT uptake.

As noted above, a quasi-experimental study found that by placing PDAs with HIV educational media in clinic waiting rooms, study respondents increased their HIV knowledge [18]. In absence of a comparison group, however, there is insufficient evidence to demonstrate that the mHealth intervention was any more effective than traditional paperbased materials and verbal messaging or patient education. Moreover, the article offers no evidence that increased HIV knowledge before clinic visits was associated with increased testing or linkage to care.

Finally, a unique pilot study by Welz and Herbst described the use of handheld computers for delivering HIV testing results in hard-to-reach South African communities [29]. As a part of a PDA-based household survey, research staff offered finger-prick testing to all adults and stored test results on secure PDAs at community-based counseling centers. Survey participants who wanted to confidentially obtain their HIV results could do so by presenting a unique code. Among those tested, 5\% received their results in this manner. The authors argue that in a traditional surveillance system, few if any would be informed of their HIV status, 


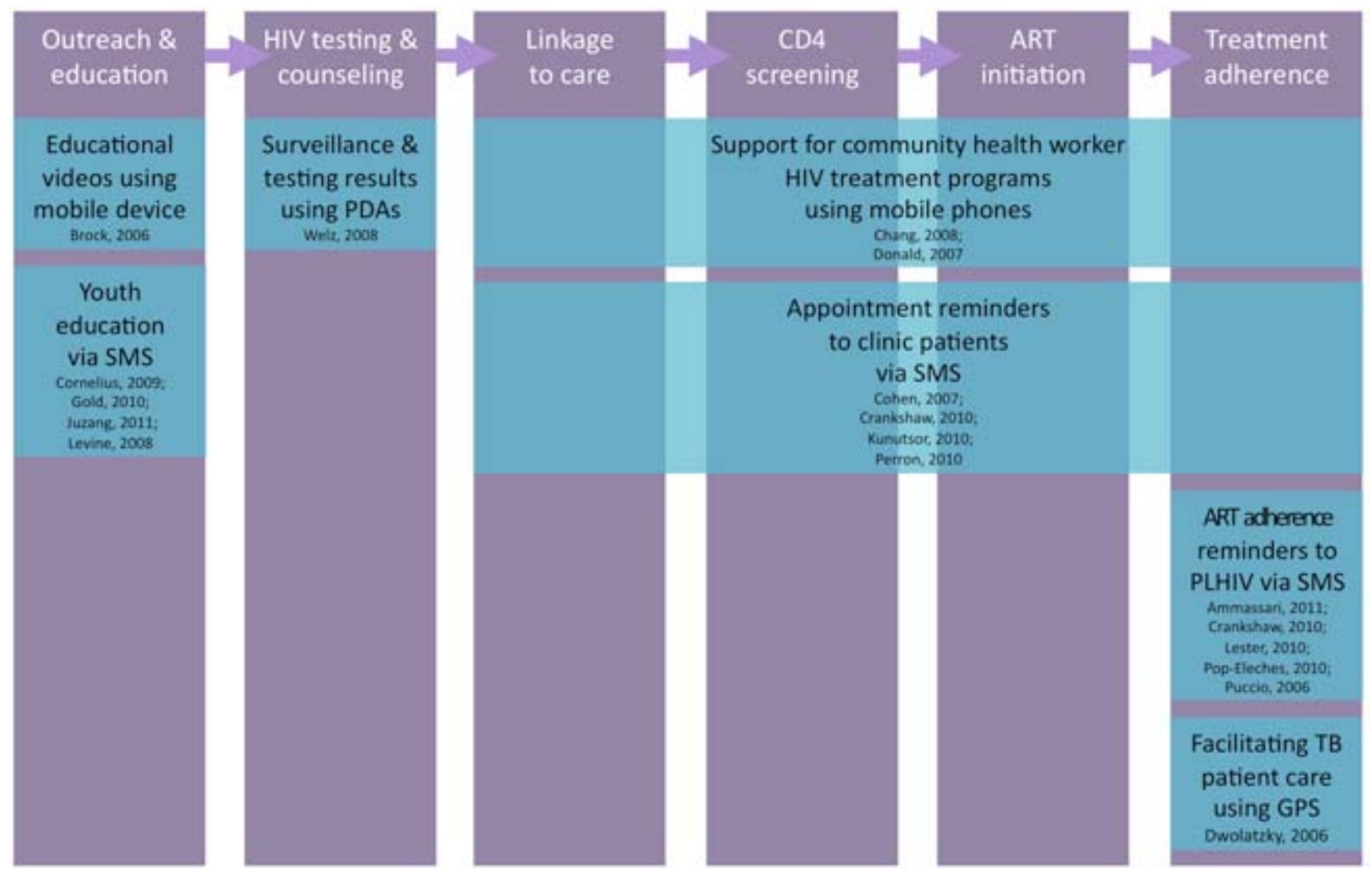

Fig. (2). mHealth evidence across the HIV treatment \& prevention cascade.

however they offer no evidence to support this claim. Although there were measures taken to protect personal data, confidentiality was not confirmed by the study.

No published articles, within the time frame of the review, described the use of mobile technologies to support counseling and screening among pregnant women and/or infants, an essential programmatic activity for the prevention of mother-to-child transmission (PMTCT) of HIV.

Linkage to Care: There is some evidence that mHealth can contribute to retention in care between testing and enrollment in HIV care. One RCT that took place in an urban European sexual health clinic found that patient reminders significantly increased appointment attendance at clinical care site [30]. The intervention, however, included text messages to mobile phones, voice phone calls, and postal reminders, making it difficult to discern the effect of mobile health tools. Simple economic analysis included in this study revealed that the reminder system paid for itself within three months because the intervention clinic was able to bill for more consultations per day.

A similar system to improve appointment attendance among PLHIV in rural Uganda was evaluated by Kunutor and colleagues [31]. In their pilot operations study, researchers assessed feasibility and program outputs associated with sending simple text messages or making a voice call to remind patients who miss their appointments to come in for a clinical visit as soon as possible. The study demonstrated that most patients who received text/voice messages presented for treatment within a few days, however the lack of comparison group and absence of multiple measurements makes it difficult to conclude that the mobile phone intervention is responsible for improved clinic attendance rates. The study included no costing details on staff time for delivering voice and text messages, which may have been helpful in comparing it to other efforts to improve retention in care.

CD4 Screening: Although point-of-care CD4 diagnostic tools may be available in the future [32] and eHealth systems have been useful for HIV patient tracking across the continuum of care [33], there is not evidence to date on the use of mHealth tools explicitly for CD4 screening. In a more generic sense, several articles [20,31,32,35-37] found that mHealth tools were helpful for managing care by improving attendance to medical appointments, during some of which one would assume CD4 testing took place, through text reminders and community health worker support. However, further detail about the use of mHealth tools for rapid, accurate, and shared CD4 results reporting was not reported explicitly, although mHealth technologies to accomplish these goals are feasible and available.

Treatment Adherence: Use of mHealth to improve ART adherence has the strongest evidence base. Several descriptive [19,34] and quasi-experimental studies [27,3537] conclude that text message reminders are acceptable, feasible, and useful for improved treatment adherence among PLHIV in resource-constrained settings. Two impact studies, both RCTs, demonstrate the effectiveness of mHealth for adherence to ART. Pop-Eleches et al. found that treatment adherence improved significantly among patients 
randomized to receive weekly text message reminders during a 48-week intervention period in Kenya [20]. The authors conclude that short weekly reminders were significantly more effective than no, daily, or longer text reminders.

Consistent with the findings Pop-Eleches et al, another Kenya-based RCT by Lester et al. found that patients receiving weekly SMS reminder messages had significantly improved antiretroviral adherence and rates of viral suppression compared with the control individuals [21]. Differing from the Pop-Eleches intervention, patients in the Lester study were instructed to respond to clinic nurses within 48 hours, confirming they received SMS. Although people enrolled in PMTCT programs were included as study participants, description of an assessment of reminders to support PMTCT, women, and mothers was not reported.

\section{DISCUSSION}

Our review reveals that the science and practice of mHealth for HIV prevention, care, and treatment are in their early stages. Although the majority of studies published focus primarily on acceptability and feasibility of early small-scale pilots, the literature reveals a promising trend toward feasible and acceptable initiatives, positive outcomes, and rigorous study design.

\section{Limitations}

mHealth is a new and rapidly evolving field, led frequently by commercial entrepreneurs and small technology non-profits. It is likely that many mHealth initiatives are not being documented or published. Efforts that lead to negative findings may be published less frequently. As a result, this review may not reflect the full mHealth landscape, particularly the unreported implementation knowledge of practitioners and institutions. Only those articles that explicitly report use of mobile information technology devices were included. As such, the review did not include articles on information communication and computer-based systems that rely on less mobile devices, including PCs, laptop computers, and printers, despite their potential relevance of these eHealth systems to mHealth. Although future reviews may find only minor differences between eHealth and mHealth tools, especially as computer-based interventions all become increasingly mobile, we found that this distinction is still relevant for the articles published in the past ten years.

\section{mHealth, HIV, \& Implementation Science}

As an emergent area of study and practice, it is not surprising that the current mHealth literature has focused mostly on assessment of acceptability and feasibility of pilot initiatives within low-resource contexts. It is likely that, as the science of mHealth matures, strategies for health systems strengthening and for addressing the HIV prevention and treatment cascade $[8,9,38]$ will increasingly incorporate mobile technologies. In this regard, development of a road map for mHealth implementation science is warranted. Implementation science is a field that explicitly aims to improve the uptake, implementation, and translation of actionable research findings into real world programs $[39,40]$ and, as such, an implementation science agenda will ultimately focus the global community on the design, development, and use of technologies that have a demonstrable impact(s).

Based on this assessment of the existing mHealth literature, there are three fundamental steps toward strengthening the implementation science evidence base. First, providing actionable evidence on how to improve HIV care through incorporation of mHealth solutions will require integration of technologies into existing programs. To date, mHealth practice is mostly characterized as small, one-off, externally driven, and isolated pilots. The literature reveals ample data related to feasibility, acceptability, and even early outcomes/impact to support the potential benefit that may accrue through larger scale implementation in the context of national HIV programs.

Second, articulation of theoretical frameworks and program logic will strengthen the mHealth evidence base. Program monitoring and evaluation requires a description of the assumptions that sequentially link inputs, activities, outputs and outcomes to intended impact(s). Although there are convincing arguments that better systems are created through user-centered design $[41,42]$, iterative development [43], and building well architected health information systems $[44,45]$, there is no evidence to demonstrate the connections between particular development processes and health outcomes. Furthermore, the literature includes some minor references to conceptual frameworks and theory, such as social action theory [46] and HIV behavior change theory [47], reports of why or how mHealth might impact individuals, social groups, institutions, or health systems were not identified in this review. mHealth interventions might benefit from the dozens if not hundreds of years of development of theories of individual, social, and institutional change, including social cognitive theory [48], unified theory of acceptance and use of technology [49], adoption of innovations [50], and others. A common set of theories of change and matrix for measurement will allow researchers to contribute to a common dialogue and build a stronger evidence-base around whether, why, and under what circumstances mHealth might improve the effectiveness and efficiencies of global HIV intervention.

Third, and as reported in the mHealth literature reviews, there remains a need to improve the rigor of mHealth research and evaluation. An implementation science agenda will drive the field towards more rigorous approaches to monitoring and evaluation, operations research, and impact evaluation [51], necessarily moving the literature from mostly descriptive studies to more quasi-experimental and experimental designs. RCTs, such as those reported by PopEleches, Lester, and their colleagues [20,21], provide strong evidence of the utility of mHealth to address the HIV prevention and treatment cascade. However, when RCTs are not feasible or ethical in practice-based contexts, implementers and researchers can push for more rigorous quasi-experimental studies [52-54] and further grounding [55] and analytical rigor [56] in qualitative studies.

Additionally, improving rigor requires expanding beyond the current trend of external expertise, leadership, and management. An implementation science agenda might also require emphasis on participatory approaches to evaluation, which have often resulted in more relevant and actionable programmatic recommendations, internal capacity building, 
and a sustainable culture of research within programs. Engaging frontline program directors, community leaders, PLHIV, and marginalized communities is critical to addressing HIV globally and practitioners of implementation science suggest that this tradition is particularly meaningful in their scientific agenda [57].

\section{mHealth \& HIV Treatment \& Prevention Programmatic Needs}

There is now evidence that mHealth tools can improve linkage to care, retention in care, and adherence to ART, however there remain some critical gaps in the evaluation and implementation of mHealth solutions. Surprisingly, there is very sparse evidence on the use of mHealth to enhance VCT uptake. The increasing saturation of mobile phones among underserved communities around the world provides an opportunity for targeted and tailored VCT outreach, education, and communications campaigns. Although evidence supports the use of eHealth systems to improve supply chain management [58-61] for HIV/AIDS and other disease issues in LMICs --such as malaria treatment [62] -the literature does not report on the promising opportunities to use mobile devices to track medications and other supplies essential for HIV service delivery at scale. Similarly, the literature on the use of eHealth systems for CD4 screening [33] has not translated to any evidence around the utility of mHealth systems.

The literature describes mHealth implementations among diverse urban and rural communities around world, however there is a lack of focus on key populations at higher risk of HIV exposure. HIV and AIDS is distinct from most other diseases and health conditions in that HIV individuals often experience lifetime stigma and discrimination [63]. Moreover, behaviors related to acquisition of HIV infection are associated with social and economic vulnerabilities [63]. Key populations -- such as pregnant mothers, sex workers, users of injection drugs, and men who have sex with men -are not only at higher risk for HIV infection, but are also less likely to access standard treatment and the other related HIV health services due to stigma and discrimination they face from the general population as well as providers [64-70]. Given the promise of mobile technology to deliver services beyond traditional clinical settings and to improve security and confidentiality of health information, greater attention to appropriately designed mHealth interventions is warranted.

Despite the feminization of HIV [71], few articles focus specifically on women and only two mention pregnant women as the target study group. One report describes a recently initiated RCT using mobile phones for data collection [72] and the other includes pregnant women in its sample but presents no findings relevant to PMTCT [21]. We found no articles that describe the use of mobile technology to support PMTCT programs, although one article on mHealth for improving efficiency of early infant diagnosis was published after the literature collection deadline [73]. The nearly complete absence of published data on mHealth for PMTCT is surprising given its priority with the global HIV-response community [74].

\section{CONCLUSION}

Our review of the evidence-based for mHealth and HIV reveals that the science and practice are evolving rapidly, but still in their early stages. Small-scale efforts, pilot projects, and preliminary descriptive studies are advancing toward increasingly systematic and rigorous monitoring/evaluation, operations research, and impact evaluation. However, there remains several gaps in an implementation science agenda. mHealth pilots must begin to scale and integrate into local and national HIV prevention and treatment programs, mHealth theoretical frameworks and program logic must be articulated and evaluated, and the overall rigor of mHealth research and evaluation must be improved to strengthen and provide actionable evidence for program leaders, policymakers, and funders.

There is evidence that mHealth tools can improve linkage to care, retention in care, and adherence to ART. Future mHealth efforts should address additional programmatic needs such as VCT uptake, PMTCT, laboratory management, and supply chain management. Most importantly, mHealth efforts must explicitly acknowledge the global focus on the most HIV-vulnerable communities, such as women, children, sex workers, MSM, and migrants.

\section{CONFLICT OF INTEREST}

The authors confirm that this article content has no conflict of interest.

\section{ACKNOWLEDGEMENTS}

This research was led by the Research and Evaluation Sub-Committee of the Health Informatics Public Private Partnership, a collaboration of the United States Agency for International Development (USAID), the Centers for Disease Control and Prevention (CDC), and the Office of the Global AIDS Coordinator (OGAC). Special thanks to institutional partners InSTEDD, mHealth Alliance, and Partners in Health. Editorial thanks to Barrot Lambdin of Pangaea Global AIDS Foundation.

\section{REFERENCES}

[1] WHO. WHO global observatory for e-health series - New horizons for health through mobile technologies. Observatory. Geneva: WHO 2011; p. 3.

[2] Tudor CL, van-Velthoven MHMMT, Brusamento S, et al. Integrating interventions for prevention of transmission of HIV from mothers to infants during pregnancy, delivery and breastfeeding with other healthcare services to increase the coverage. Cochrane Database Syst Rev (Online). 2011; (6): CD008741.

[3] Bailey R, Moses S, Parker C, Agot K. Male circumcision for HIV prevention in young men in Kisumu, Kenya: a randomised controlled trial. Lancet 2007; 369(9562): 643-56.

[4] Gray R, Kigozi G, Serwadda D. Male circumcision for HIV prevention in men in Rakai, Uganda: a randomised trial. Lancet 2007; 369(9562): 657-66.

[5] Baeten J, Celum C. Antiretroviral pre-exposure prophylaxis for HIV-1 prevention among heterosexual African men and women: the Partners PrEP study. IAS Conference on HIV Pathogenesis, Treatment and Prevention. Rome; 2011.

[6] UNAIDS. Getting to zero: 2011-1015 Strategy. Development. Geneva: UNAIDS 2010.

[7] Clinton HR. Remarks on "Creating an AIDS-Free Generation". Bethesda: National Institute of Health 2011.

[8] El-sadr WM, Affrunti M, Gamble T, Zerbe A. Antiretroviral Therapy : a promising HIV prevention strategy ? J Acquir Immune Defic Syndr 2010; 55:116-21. 
[9] Micek MA, Gimbel-Sherr K, Baptista AJ, et al. Loss to follow-up of adults in public HIV care systems in central Mozambique: identifying obstacles to treatment. J Acquir Immune Defic Syndr (1999) 2009; 52(3): 397-405.

[10] Rosen S, Fox MP. Retention in HIV care between testing and treatment in sub-Saharan Africa: a systematic review. Bartlett J, Ed. PLoS 2011; 8(7): e 1001-56.

[11] Kranzer K, Lewis JJ, Ford N, et al. Treatment interruption in a primary care antiretroviral therapy program in South Africa : cohort analysis of trends and risk factors. J Acquir Immune Defic Syndr 2010;55(3):17-23.

[12] Chiasson MA, Hirshfield S, Rietmeijer C. HIV prevention and care in the digital age. J Acquir Immune Defic Syndr 2010; 55(Suppl. 2).

[13] mHealth Alliance. What is mHealth?Available at: www.mhealthalliance.org 2012.

[14] Torgan C. The mHealth summit: local \& global converge. Washington D.C; 2009.

[15] WHO. M-Health. Geneva: WHO 2013

[16] Blaya J. E-health technologies show promise in developing countries. Health Aff 2010; 29(2): 244-51.

[17] Kho A, Henderson LE, Dressler DD, Kripalani S. Use of handheld computers in medical education. A systematic review. J Gen Intern Med 2006; 21(5):531-7.

[18] Marcus J, Wong Kromhout W. NIH recognizes engineering professor's innovative research with major award: pioneering work on cell-phone imaging could transform global health care. Los Angeles: UCLA Newsroom 2009

[19] Brock TP, Smith SR. Using digital videos displayed on personal digital assistants (PDAs) to enhance patient education in clinical settings. Int J Med Inform 2007; 76(11-12): 829-35.

[20] Crankshaw T, Corless IB, Giddy J, Nicholas PK, Eichbaum Q, Butler LM. Exploring the patterns of use and the feasibility of using cellular phones for clinic appointment reminders and adherence messages in an antiretroviral treatment clinic, Durban, South Africa. AIDS Patient Care STDS 2010; 24(11):729-34.

[21] Pop-Eleches C, Thirumurthy H, Habyarimana JP, et al. Mobile phone technologies improve adherence to antiretroviral treatment in a resource-limited setting: a randomized controlled trial of text message reminders. AIDS (London, England) 2011; 25(6):825-34.

[22] Lester RT, Ritvo P, Mills EJ, et al. Effects of a mobile phone short message service on antiretroviral treatment adherence in Kenya (WelTel Kenya1): a randomised trial. Lancet 2010; 376(9755):1838-45

[23] Gold J, Lim MSC, Hellard ME, Hocking JS, Keogh L. What's in a message? delivering sexual health promotion to young people in Australia via text messaging. BMC Public Health 2010; 10: 792.

[24] Samal L, Hutton HE, Erbelding EJ, Brandon ES, Finkelstein J, Chander G. Digital divide: variation in internet and cellular phone use among women attending an urban sexually transmitted infections clinic. Bull NY Acad Med J Urban Health 2010; 87(1):122-8.

[25] Swendeman D, Rotheram-Borus MJ. Innovation in sexually transmitted disease and HIV prevention: Internet and mobile phone delivery vehicles for global diffusion. Curr Opin Psychiatry 2010; 23(2):139-44.

[26] Tolly K De, Alexander H, Town C. Innovative use of cellphone technology for HIV / AIDS behaviour change communications : 3 pilot projects Cellphones4 HIV project SMS ARV reminders : TAC adherence clubs. Cell 2009;(May 2008):1-13.

[27] Vidrine DJ, Arduino RC, Gritz ER. Impact of a cell phone intervention on mediating mechanisms of smoking cessation in individuals living with HIV/AIDS. Nicotine Tobacco Res 2006; 8 Suppl 1: S103-8.

[28] Levine D, McCright J, Dobkin L, Woodruff AJ, Klausner JD. SEXINFO: a sexual health text messaging service for San Francisco youth. Am J Public Health 2008; 98(3): 393-5.

[29] Welz T, Herbst K. Anonymous HIV testing with participantcontrolled access to results using handheld computers: a new model of HIV testing used in a household survey in rural South Africa. Sex Transm Dis 2008; 35(4): 372-6.

[30] Perron NJ, Dao MD, Kossovsky MP, et al. Reduction of missed appointments at an urban primary care clinic: a randomised controlled study. BMC Fam Pract 2010;11(79):p.8.

[31] Kunutsor S, Walley J, Katabira E, et al. Using mobile phones to improve clinic attendance amongst an antiretroviral treatment cohort in rural Uganda: a cross-sectional and prospective study. AIDS Behav 2010;14(6):1347-52

[32] Marcus J, Wong Kromhout W. NIH recognizes engineering professor's innovative research with major award: pioneering work on cell-phone imaging could transform global health care. Los Angeles: UCLA Newsroom 2009.

[33] Fraser HSF, Allen C, Bailey C, Douglas G, Shin S, Blaya J. Information systems for patient follow-up and chronic management of HIV and tuberculosis: a life-saving technology in resource-poor areas. J Med Intern Res 2007; 9(4): e29.

[34] Chang LWLW, Kagaayi J, Nakigozi G, et al. Letter to the Editor: Responding to the human resource crisis: peer health workers, mobile phones, and HIV care in Rakai, Uganda. AIDS patient care and STDs. Mary Ann Liebert, Inc. 140 Huguenot Street, 3rd Floor New Rochelle, NY 10801-5215 USA 2008; 22(3):173-4.

[35] Donald S, Ulrike R, Charissa B, Skinner D. Evaluation of use of cellphones to aid compliance with drug therapy for HIV patients. Aids Care-Psychol Socio-Med Asp Aids/HIV 2007; 19(5): 605-7.

[36] Cohen CE, Coyne KM, Mandalia S, Waters AM, Sullivan AK. Time to use text reminders in genitourinary medicine clinics. Int $\mathrm{J}$ STD AIDS 2008; 19(1):12-3.

[37] Shet A, Arumugam K, Rodrigues R, et al. Designing a mobile phone-based intervention to promote adherence to antiretroviral therapy in South India. AIDS Behav 2010;14(3): 716-20.

[38] Ammassari A, Trotta MP, Shalev NM, et al. Timed short messaging service improves adherence and virological outcomes in HIV-1-infected patients with suboptimal adherence to antiretroviral therapy. J Acquir Immune Defic Syndr 2011; 58(4):e113-5.

[39] Micek M, Gimbel-Sherr K, João Baptista A, et al. Mozambique : Identifying obstacles to treatment. J Acquir Immune Defic Syndr 2010; 52(3): 397-405.

[40] Madon T, Hofman KJ, Kupfer L, Glass RI. Implementation Science. Science 2008; 318:1728-9.

[41] The global fund to fight AIDS tuberculosis and malaria, United States Agency for International Development (USAID), World Health Organization (WHO), special program for research and training in tropical diseases JUNP on H (UNAIDS), The World Bank. Framework for Operations and Implementation Research in Health and Disease Control Programs 2008.

[42] Dabbs ADV, Myers BA, Mc Curry KR, et al. User-centered design and interactive health technologies for patients. Comput Inform News 2009; 27(3):175-83.

[43] Norman D. The design of everyday things. New York: Basic Books 1988.

[44] Nielsen J. Iterative user interface design. IEEE Compute 1993; 26(11):32-41.

[45] Jones DT, Duncan R, Langberg ML, Shabot MM. Technology architecture guidelines for a health care system. Proceedings AMIA Symp 2000; pp. 399-402.

[46] Stansfield S, Orobaton N, Lubinski D, Uggowitzer S, Elec BE, Mwanyika $\mathrm{H}$. The case for a national health information system architecture ; a missing link to guiding Nat Dev Implementation. Belagio 2009; pp. 1-9.

[47] Ewart C. Social action theory for a public health psychology. Am Psychol 1991;(46): 931-46.

[48] Rotheram-Borus M-J, Richter L, Van Rooyen H, et al. Project masihambisane: a cluster randomised controlled trial with peer mentors to improve outcomes for pregnant mothers living with HIV. Trials 2011;12: 2 .

[49] Bandura A. Social cognitive theory: an agentic perspective. Ann Rev Psychol 2001; 52:1-26.

[50] Lee Y, Kozar KA, Larsen KRT. The technology acceptance model : past, present, and future. Commun Assoc Inf Syst 2003 , 12(1): 752-80.

[51] Rogers EM. Diffusion of innovations. Glencoe: Free Press; 1962.

[52] Padian NS, Holmes CB, Mccoy SI, Lyerla R, Bouey PD, Goosby EP. Implementation science for the US president' $s$ emergency plan for AIDS relief ( PEPFAR ). J Acquir Immune Defic Syndr 2011; 56(3):199-203.

[53] Handley MA, Schillinger D, Shiboski S. Quasi-Experimental designs in practice-based research settings: design and implementation considerations. J Am Board Fam Med 2011; 24(5): 589-96.

[54] Bonell CP, Hargreaves J, Cousens S, et al. Alternatives to randomisation in the evaluation of public health interventions: 
design challenges and solutions. J Epidemiol Commun Health 2011; 65(7): 582-7.

[55] Hussey MA, Hughes JP. Design and analysis of stepped wedge cluster randomized trials. Contemp Clin Trials 2007; 28(2):182-91.

[56] Corbin JM, Strauss AL. Basics of qualitative research: techniques and procedures for developing grounded theory. $3^{\text {rd }}$ ed. Thousand Oaks, CA: Sage Publications, Inc.; 2008; p. 379.

[57] Pope C, Ziebland S, Mays N. Qualitative research in health care. Analysing qualitative data. BMJ 2000; 320(7227):114-6.

[58] Swaminathan S, Guay L. How to conduct operational research and implementation science - definitions, design, Methodology. 6th International AIDS Society Conference on HIV Pathogenesis, Treatment and Prevention. Rome; 2011.

[59] Berger EJ, Jazayeri D, Sauveur M, et al. Implementation and evaluation of a web based system for pharmacy stock management in rural Haiti. AMIA: Annual Symposium proceedings / AMIA Symposium. AMIA Sympos 2007; pp.46-50.

[60] Nugent C, Finlay D, Black N. Mobile management and prescription of medication. M-Health 2006.

[61] Dass R, Gajjar B. Anti-counterfeit technologies for spurious drugs in India. Int J User-Driven Healthcare 2011;1(4).

[62] Woudhuysen J, Rivers P. Rethinking packaging: how electronic packs for pharmaceuticals work with mobile IT to improve patient adherence to medication regimens. Smart Des 2012; pp.1-11.

[63] Barrington J, Wereko-Brobby O, Ward P, Mwafongo W, Kungulwe S. SMS for life: a pilot project to improve anti-malarial drug supply management in rural Tanzania using standard technology. Malar J 2010; 9(298):1-9.

[64] Mahajan AP, Sayles JN, Patel VA, et al. Stigma in the HIV/AIDS epidemic: a review of the literature and recommendations for the way forward. AIDS (London, England) 2008; 22 Suppl 2: S67-79.
[65] Niang C, Tapsoba P. "It"s raining stones': Stigma, violence and HIV vulnerability among men who have sex with men in Dakar, Senegal. Culture, Health Sex 2003; 5(6): 499-512.

[66] PEPFAR. Addressing gender and HIV/AIDS. Washington, D.C: PEPFAR 2010.

[67] Castro A, Farmer P. Understanding and addressing AIDS-related stigma: from anthropological theory to clinical practice in Haiti. J Inf 2005; 95(1):53-9.

[68] Ogden J, Nyblade L. Common at its core: HIV-related stigma across contexts. Washington, D.C: ICRW 2005

[69] UNAIDS. HIV-related stigma, discrimination and human rights violations: Case studies of successful programmes. Geneva: UNAIDS 2005.

[70] Farmer P, Connors M. Women, poverty, and AIDS: sex, drugs, and structural violence. $2^{\text {nd }}$ ed. Monroe: Common Courage Press 2011.

[71] Parker R. The global HIV/AIDS pandemic, structural inequalities and the politics of international health. Am J Public Health 2002; 92(3): 343-6.

[72] Quinn TC, Overbaugh J. HIV/AIDS in women: an expanding epidemic. Science 2005; 308(5728):1582-3.

[73] Rotheram-Borus M-J, Richter L, Van Rooyen H, et al. Project masihambisane: a cluster randomised controlled trial with peer mentors to improve outcomes for pregnant mothers living with HIV. Trials 2011; 12(2):1-10.

[74] World Health Organization (WHO). Antiretroviral drugs for treating pregnant women and preventing HIV infections in infants: Recommendations for a public health approach. Geneva: WHO 2010.

(c) Catalani et al.; Licensee Bentham Open.

This is an open access article licensed under the terms of the Creative Commons Attribution Non-Commercial License (http://creativecommons.org/licenses/by-nc/ $3.0 /$ ) which permits unrestricted, non-commercial use, distribution and reproduction in any medium, provided the work is properly cited. 